\title{
THE EYE OF THE BEHOLDER: DESIGNING THE OWL
}

\author{
P. Dierickx, J. L. Beckers, E. Brunetto, R. Conan, E. Fedrigo, R. Gilmozzi, \\ N. Hubin, F. Koch, M. Le Louarn, E. Marchetti, G. Monnet, L. Noethe, \\ M. Quattri, M. Sarazin, J. Spyromillo, N. Yaitskova
}

European Southern Observatory

\begin{abstract}
Preliminary requirements and possible technological solutions for the next generation of ground-based optical telescopes were laid down at ESO in 1998. Since then, a phase A study has been commissioned, the objective of which is to produce a conceptual design compatible, to the maximum possible extent, with proven technology, and establish realistic plans for detailed design, site selection, construction and operation for a 100-m class optical, diffractionlimited telescope. There was no doubt about how daunting such a challenge would be, but, somewhat surprisingly, it turns out to be firmly confined to adaptive optics concepts and technologies. The telescope itself appears to be feasible within the allocated budget and without reliance on exotic assumptions. Fabrication of key subsystems is fully within the reach of a properly engineered, industrialized process. A consolidated baseline is taking shape, and alternative system and subsystem solutions are being explored, strengthening the confidence that requirements could be met. Extensive development of wavefront measurement techniques enlarges the palette of solutions available for active wavefront control of a segmented, active telescope. At system level, ESO is developing enabling experiments to validate multi-conjugate adaptive optics (MAD for Multi-conjugate Adaptive optics Demonstrator) and telescope wavefront control (APE, for Active Phasing Experiment).
\end{abstract}

Keywords: Extremely Large Telescopes, OWL, adaptive optics, phasing, astronomical site selection.

\section{INTRODUCTION}

The European Southern Observatory (ESO) presented early feasibility considerations for a 100-m optical telescope in $1998^{1}$. Although proposals for telescopes with diameters much larger than deemed possible had been laid down as early as $1978^{2,3}, 1992^{4}$ and $1996^{5}$, this was, perhaps, the first occasion where not only possible design solutions but also plausible fabrication technologies were introduced. Since then, the field of Extremely Large Telescopes has rapidly expanded, with concepts of 20- to 100-m telescopes being promoted worldwide.

We identify three factors that support such unprecedented increase in telescope diameter: optical segmentation, active wavefront control, adaptive optics. Optical segmentation, demonstrated in the Keck and Hobby-Eberly, eliminates the traditional limit imposed by the slowly evolving processes of producing large, homogeneous optical subtrates. With spherical mirror designs, the same applies to polishing. Active wavefront control, which we define as the ability to monitor and control telescope errors in real time -as demonstrated in the NTT and deployed on VLT, Gemini, Subaru, and Magellan, guarantees optimal performance while allowing substantial relaxation of fabrication and stiffness tolerances. Finally, adaptive optics allows the geometrical extent to remain compatible with realistic instrumentation and, last but not least, dramatically increases optical efficiency by freeing the telescope from the adverse effect of atmospheric turbulence. Indeed, the peak signal $\mathrm{S}$ in a long-exposure, seeing-limited Point Spread Function (PSF) is ${ }^{6}$ $S \propto \tau I r_{0}^{2} D^{2}$, where $\tau$ is the transmission efficiency, $I$ the Central Intensity Ratio (CIR, or normalized Strehl Ratio), $r_{0}$ the atmospheric coherence length and $D$ the aperture diameter. In diffraction-limited regime, the relation becomes $S$ $\propto \tau R D^{4}$, where $\mathrm{R}$ is the Strehl Ratio. The gain implied by adaptive optics is therefore proportional to $\left(D / r_{0}\right)^{2}$. With a 100 -m telescope and good seeing, it is $\sim 10^{4}$ at $2.2 \mu \mathrm{m}$ and about $2.5 \times 10^{5}$ in the visible. Typical values of the CIR and Strehl Ratio will not change the figure dramatically. We conclude that not only do segmentation, active and adaptive optics make extremely large telescopes feasible, the latter makes them extremely attractive in terms of scientific productivity. Of all three techniques, this is also the only one whose extrapolation beyond existing apertures requires substantial development. This is, arguably, also the reason why the distinction between active and adaptive optics will most likely persist over OWL timescale but, perhaps, not beyond. 
Early work on the OWL concentrated on optical fabrication of the primary mirror, now widely regarded as a non-issue -although there is disagreement in the perception of performance, risk, schedule and cost implied by mirror shape (spherical or aspherical). Meanwhile, designs explored worldwide appeared to follow a clear polarization: 30-m (CELT and GSMT to name the most advanced ones) vs. the OWL 100-m concepts with different design approaches. The former rely on a maximum extrapolation of traditional optical designs, while the latter aims at the limit permitted by available technology within a fixed cost target, the opto-mechanical design being allowed to depart from traditional solutions to allow such limit to be safely attained. The resulting concepts have often been compared on the assumption that traditional Ritchey-Chrétien designs would automatically meet requirements while fabrication issues -in particular the polishing and testing of off-axis aspherical segments- could be solved at an affordable cost. Such assumption may, perhaps, still be acceptable in the 30-m range, but certainly not in the 100-m one. The 30- to 100-m gap could be filled by the 50-m EURO-507 , but its design does not have the simplicity of the 30-m Ritchey-Chrétien ones and is making more ambitious assumptions as to the timely feasibility of its aspherical optics. Last but not least, the comparison between various concepts cannot be limited to fabrication issues, as other and eventually more essential system considerations must be taken into account ${ }^{8}$, not to mention scientific ones, which fall outside the scope of this article.

The need of competitive schedule and cost plays a major role in the design of OWL. The design relies on high redundancy and readily available, industrialized processes -e.g. all-identical spherical segments for the primary mirror, structure assembled from nearly all-identical modules, etc. Paradoxically, the sheer number of components to be made and assembled allows attractive cost-schedule trade-offs. A case in point is, again, the main optics. In the case of the VLT, investments in production facilities represented the largest fraction of the total cost. With OWL, they amount to $10-20 \%$ according to industrial estimates. Increased production capability is therefore not a major cost constraint. The situation is even more favorable with the mechanical modules, whose production and assembling do not require specialized facilities and can be procured from several suppliers if need be. Taking this into account, it appears technically feasible to design and build the telescope structure within a reasonably short time. Industrial studies confirm that the selected fabrication processes would allow the telescope to start science operations in 2012 with a 45-m aperture, in 2013 with a 70-m one and in 2015 with full capacity. This estimate also takes into account a conservative roadmap towards large (2- to eventually 4-m) adaptive mirrors and does not require major investment before 2007. It is not what technology, unlimited resources and readily available funding would allow, but the outcome of a cautiously optimistic plan.

Other major design drivers include low sensitivity to external loads and the ability to perform adequate wavefront control functions prior to adaptive correction, so as not to overload the most challenging subsystems with an undesirably high correction range.

The baseline optical design is a folded version of that of the Hobby-Eberly telescope, with spherical primary and flat folding secondary, both segmented. A four-elements corrector provides a well-corrected, 10 arc minute field of view. The tertiary and quaternary mirrors (8-m thin monolithic) are suitably located for active optics. Assuming that large adaptive mirror technology could be extrapolated from the current 1-m LBT ${ }^{9}$ to 2.4- and eventually 4-m, the M6 and M5 mirrors of the corrector would allow near-IR adaptive optics at 0 and $7.8 \mathrm{~km}$ conjugates. These adaptive mirrors would also serve for first-stage correction for Extreme Adaptive Optics (XAO), thereby enlarging the palette of acceptable MEMs technologies for the high spatial frequency correction.

The design of the structure and kinematics has progressed ${ }^{10,11}$ towards further mass reduction and cost-saving, while the performance, as measured by the first locked rotor eigenfrequency, has been kept within a safe limit of $2 \mathrm{~Hz}$. Dynamic analysis has been performed to evaluate the performance of the structure and set requirements for the control systems, and we have started to plan for an end-to-end model. Detailed diffraction models ${ }^{10}$ have been developed to assess the impact of segmentation and segments edge misfigure on the scientific performance of the telescope, particular attention being paid to imaging of exoplanets.

Several industrial studies have already been completed, most notably in the area of mass-produced optical substrates and critical mechanical elements. Four Silicon Carbide segment blanks prototypes are supplied by ASTRIUMBOOSTEC, with a view to qualifying this technology for the segments of OWL as a valid alternative to the baseline glass-ceramics. Fluid dynamics simulations are currently running to evaluate wind disturbances, with encouraging first results $^{12}$. These simulations, to be completed by wind tunnel testing as well as possible measurements on the 
Effelsberg 100-m radio telescope, provide input for design iterations and wavefront control requirements. Future studies will shift the emphasis towards segment polishing, actuators, sensors, telescope metrology and kinematics, control systems, to name a few.

In parallel to the telescope design and industrial studies, substantial effort is also put on adaptive optics (AO). Models are being implemented to eventually allow full-scale simulations ${ }^{13}$, while early calculations confirm the substantial gain in sky coverage implied by the telescope aperture ${ }^{14}$. Evaluations of the effect of the outer scale of atmospheric turbulence yield encouraging results, not the least surprising one being that with OWL, diffraction-limited resolution may be achievable without adaptive correction ${ }^{13}$. Novel approaches towards multi-conjugate adaptive optics are also explored -mainly through an EU-funded Research \& Training Network. The Network is also undertaking a review of possible phasing techniques ${ }^{15,16}$.

A worldwide search for a suitable site is under way. So far no preference to a given hemisphere has emerged from the science case. The ESPAS (ESO's Search for Potential Astronomical Sites) Group is undertaking a comprehensive review of known sites, some of which deemed as possible candidates for OWL. Quantifying the properties of such sites, understanding and modeling them, may eventually provide relevant criteria to search databases and allow to identify yet unknown candidates. Under contract with the University of Fribourg, ESO is developing tools to screen ground stations and satellite databases ${ }^{17}$ for such criteria.

\section{TOP LEVEL REQUIREMENTS}

The top-level requirements are outlined in table 1. Emphasis is put on the telescope aperture and subsequent resolution in adaptive mode, sensitivity gain being proportional to the fourth power of the aperture instead of its square in seeinglimited conditions, as discussed in the introduction.

It is expected that the scientifically useful field of view, including that devoted to finding suitable references for adaptive optics, will only exceptionally exceed 6 arc minutes. Outer areas (beyond 6 arc minutes and up to 10 arc minutes diameter) of the technical field should provide suitable references for active optics and occasional phasing calibrations, without overheads. It should be noted that for science cases calling for resolution of $\sim 0.1$ to 0.001 arc seconds, a science field of a few arc minutes generally implies a massive amount of data. After adaptive optics, efficient sampling and processing of such vast amount of information may represent OWL's second most challenging endeavor.

The matter of sky coverage, not listed in table 1 , is yet to be clarified. It seems that most if not all science cases could be addressed with a sky coverage that would appear modest with classical telescopes. Taking into account the favorable geometry implied

\begin{tabular}{|c|c|c|}
\hline \multicolumn{3}{|c|}{ Outline of top level requirements (tentative) } \\
\hline Pupil size (diameter) & 100 & $\mathrm{~m}$ \\
\hline Collecting area & $>6000$ & $\mathrm{~m}^{2}$ \\
\hline \multicolumn{3}{|c|}{ Multi-conjugate AO with Natural or Laser Guide stars } \\
\hline \multicolumn{3}{|c|}{ Diffraction-limited resolution over field of view FOV: } \\
\hline Visible $(0.5 \mu \mathrm{m})$ & $\mathrm{FOV}>30$ & arc seconds \\
\hline Infrared $(2 \mu \mathrm{m})$ & $\mathrm{FOV}>2$ & arc minutes \\
\hline \multicolumn{3}{|l|}{ Strehl ratio (at $0.5 \mu \mathrm{m}$ ) } \\
\hline Requirement & 20 & $\%$ \\
\hline Goal & 30 & $\%$ \\
\hline Seeing-limited field of view & 10 & arc minutes \\
\hline Wavelength range & $0.32-12$ & $\mu \mathrm{m}$ \\
\hline \multicolumn{3}{|l|}{ Elevation range } \\
\hline Operational & $30-89$ & degrees \\
\hline Technical & $0-90$ & degrees \\
\hline Maximum cost & 1000 & $\mathrm{M} €$ \\
\hline
\end{tabular}
by the large aperture, in particular the substantial

Table 1. Outline of top-level requirements overlap of beam footprints at high altitudes, together with the promises of multiple-field adaptive optics, sky coverage with Natural Guide Stars should not be an issue in the infrared, down to the galactic poles ${ }^{14}$. The situation is less clear in the visible, where much exploratory work is still required to lay down possible Laser Guide Stars requirements.

The current design meets all goals, least to say requirements. The cost ceiling is a particularly strong one, substantial cost overrun implying a reduction of telescope aperture, with a loss of efficiency commensurate with the fourth power law of diameter detailed in the introduction.

Design guidelines and engineering requirements emphasize reliance on proven and reliable fabrication and integration solutions. Design guidelines favor solutions that have the lowest sensitivity to inevitable error sources, such as gravity, 
wind load, thermal changes, etc. Predictability is also a strong driver for the current design, fabrication and integration plans. The plan underlying the current schedule estimate includes a number of validating experiments (briefly discussed later on), subsystems competitive preliminary designs, breadboards and prototypes.

\section{OPTICAL DESIGN AND PROPERTIES}

Several opto-mechanical concepts have been explored ${ }^{18}$, from classical Ritchey-Chrétien to siderostat solutions. The optical design converged quite rapidly towards spherical primary mirror solutions, aspherical designs being found to imply higher cost, schedule and performance risks, and substantially tighter structural requirements without providing commensurate system advantages. In addition to the arguments already exposed in the literature ${ }^{8,19}$, it should be noted that the optical design of OWL allows transferring tight centering tolerances from a 100-m scale (that of the telescope) to a $\sim 20-\mathrm{m}$ one (that of the corrector), as will be shown later. This is extremely beneficial to cost and performance, as it reduces the need for shielding from wind excitation, and relaxes correction requirements for active subsystems.

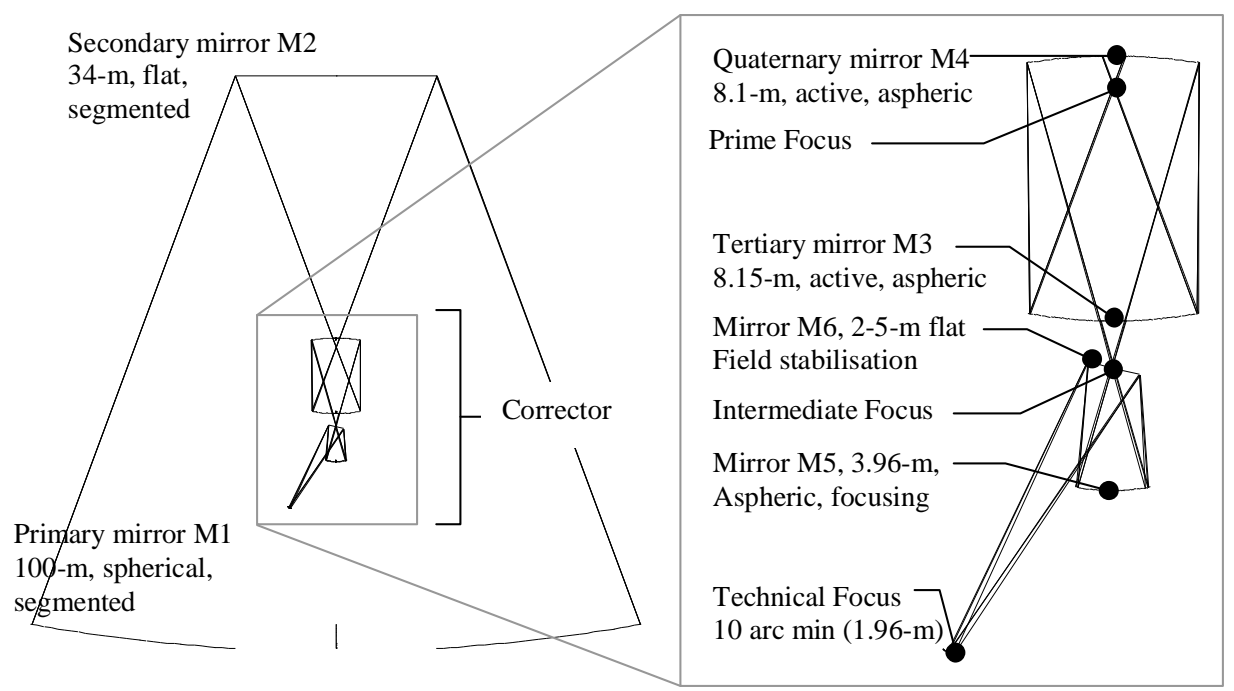

Fig. 1. Owl optical design layout

Nominal optical design data are given in table 2. The difference between physical and useful diameters corresponds to bevels. Useful diameters refer to the polished surfaces; M2 and M4 are slightly oversized for laser guide star imaging.

Diaphragms listed in table 2 can be considered as fictive surfaces and may serve no other purpose than providing conveniently located position references. Diameters are calculated with real rays; paraxial ray trace may lead to slightly different values. M4 and M6 coincide with pupil images. The design is evidently favorable to suitable baffling and simple concepts have already been explored. The deviations of M3, M4 and M5 from best fitting sphere are $0.21,9.09$, and $0.30 \mathrm{~mm}$, respectively. Generating the highly aspherical shape of the quaternary mirror is quite a challenge, but a suitable -although rather complex- test set-up which guarantees proper matching has been identified and fabrication tolerances have been found to comply with the technology used to figure the VLT primary mirrors ${ }^{16}$. Implied schedule constraints are fully compatible with OWL plan.

The as-designed optical quality fulfills the goals, with diffraction-limited quality over 3 arc minute in the visible and fully seeing-limited performance $(\sim 0.07$ arc seconds RMS) over the entire field of view. Fig. 2 shows the Strehl Ratio at visible and infrared wavelengths; the

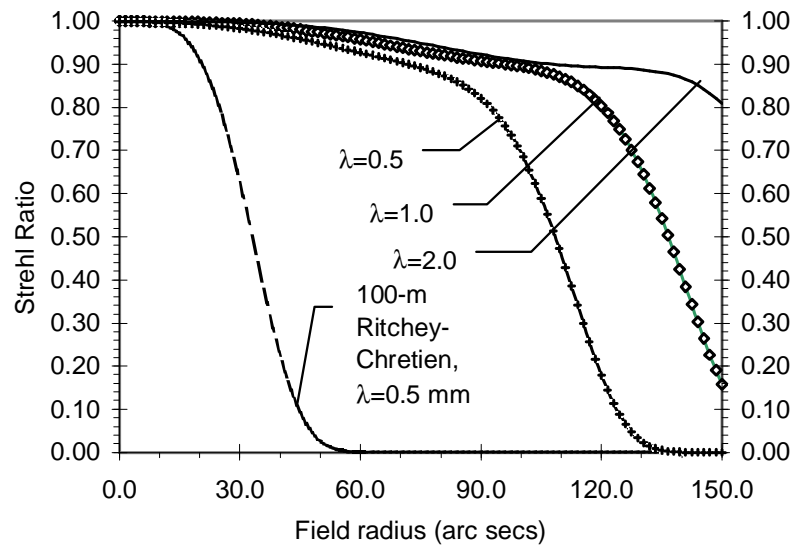

Fig. 2. Strehl ratio (curved focal surface). 
performance of a 100-m Ritchey-Chrétien design with comparable primary-secondary mirrors separation is shown for reference.

The optical properties of the design in terms of imaging of Laser Guide Stars (LGS) and atmospheric layers have been explored in depth ${ }^{20}$. The unsurprising conclusion is that the limited distance of such reference implies severe aberrations, and prevents not only focus and tilt to be adaptively compensated in closed-loop on LGS, but also third order Seidel aberrations. These modes will only be controllable with Natural Guide Stars (NGS), but their low spatial frequency translates into generous sub-pupil size hence rather faint sources. The imaging path for LGS must nevertheless be compensated for low order terms as a function of zenithal distance and altitude of the Sodium layer, to relaxed accuracy as these modes need only to be compensated in the LGS metrology path. Novel ideas as to the use of LGS for wavefront sensing may provide an alternative solution in the form of PIGS (Pseudo-Infinite Guide Stars), whereby the light beams coming from elongated and aberrated references are spatially selected to retrieve the phase error as if the beams were coming from a source at infinite distance ${ }^{21}$.

\begin{tabular}{|c|c|c|c|c|c|c|c|c|}
\hline \multirow[b]{2}{*}{ No } & \multirow[b]{2}{*}{ Component } & \multirow{2}{*}{$\begin{array}{l}\text { Radius of } \\
\text { curvature }\end{array}$} & \multirow{2}{*}{$\begin{array}{c}\text { Distance } \\
\text { to next } \\
\text { surface }\end{array}$} & \multicolumn{2}{|c|}{ Useful diameter } & \multicolumn{2}{|c|}{ Physical diameter } & \multirow[b]{2}{*}{ Remark } \\
\hline & & & & Inner & Outer & Inner & Outer & \\
\hline 1 & M1 & 284000.0 & 95000.00 & 33000 & 100000 & TBD & TBD & Entrance pupil \\
\hline 2 & M2 & Infinity & 43320.00 & 10640 & 33400 & TBD & TBD & \\
\hline 3 & Diaphragm & Infinity & 12425.00 & - & 1900 & - & TBD & $\begin{array}{l}\text { Coincides with M4 } \\
\text { vertex }\end{array}$ \\
\hline 4 & M3 & 22400.0 & 12425.00 & 1680 & 8150 & 1676 & 8200 & Concave aspheric \\
\hline 5 & M4 & 22750.0 & 12425.00 & 2680 & 8100 & 2640 & 8200 & Concave aspheric \\
\hline 6 & Diaphragm & Infinity & 2200.00 & - & 1676 & - & TBD & $\begin{array}{l}\text { Coincides with M3 } \\
\text { vertex }\end{array}$ \\
\hline 7 & Diaphragm & Infinity & 5680.00 & - & 670 & - & TBD & $\begin{array}{l}\text { Coincides with M6 } \\
\text { vertex }\end{array}$ \\
\hline 8 & M5 & 8870.0 & 5680.00 & 336 & 3960 & 306 & 3980 & Concave aspheric \\
\hline 9 & M6 & Infinity & 13491.30 & $754 \times 768$ & $2270 \times 2528$ & $750 \times 764$ & $2274 \times 2532$ & Flat, elliptical \\
\hline 10 & Image & 2472.13 & 0.00 & - & 1959 & - & 1959 & Convex, conic \\
\hline
\end{tabular}

Table 2. Nominal optical design data (technical focus). TBD is meant for To Be Determined.

Sensitivity to external loads is an essential design driver, decenters approximately increasing with the square of the telescope length under inevitable loads such as gravity and wind. This is yet another reason for OWL design to depart from classical aspherical primary mirror solutions. First, the need for a short structure implies a fast focal ratio of the primary mirror, thereby exacerbating fabrication issues and centering tolerances. Second, the surface most sensitive to decenters -the secondary mirror- is at a location where flexions are maximal. With OWL, the problem has been somewhat transferred from a 95-m scale (the separation of the primary to secondary mirrors) to a 20-m one (the dimensions of the corrector). Under a given load the displacement of the corrector is about 6 times lower than that of the secondary mirror (M2) that, being flat, impairs performance through tilt, and to a lower extent, piston only. As for the latter, the optical prescription can be fully restored by a rotation of the corrector about the vertex of M2. There is, therefore, no need to relocate the secondary mirror at any time. Active centering of the corrector may either rely on internal metrology or on-sky wavefront sensing; a detailed trade-off remains to be performed. Tolerances have yet to be established and will depend on the active optics control strategy; in the worst case figure the corrector would have to be actively aligned within a few arc seconds of its ideal position. With the current design of the structure the range of active tip-tilt of the corrector about the vertex of M2 is expected to remain within $\sim 3$ arc minutes, i..e. a very small value for a structure of OWL's dimensions.

Centering tolerances inside the corrector are more demanding, but there is ample design space for a structure even much stiffer than that of a telescope of similar size.

Sensitivity to decenters is shown in Fig. 3. The left side shows the overall effect of unit decenters, the right one the field-dependent ones only i.e. those that could not be compensated by an active deformation of the quaternary mirror. The figures clearly indicate that there is little concern about the implied accuracy of active centering mechanisms.

Optical fabrication aspects have been already exposed at length in the literature ${ }^{1,8,19}$ and will not be recalled here. It should however be mentioned that industrial studies under ESO contracts with Astrium-Boostec for Silicon Carbide segment blanks, Zeiss-LZOS, SCHOTT and Corning for glass and glass-ceramics ones, confirm the timely feasibility 
of the main optics, with a typical production cycle of $\sim 6$ years after a $\sim 2-3$ years setting up of production facilities. Cost estimates are more contrasted, some but not all figures obtained from potential vendors being within the expectations of the authors. It shall be noted that Silicon Carbide appears as a serious contender; and 1-m class prototypes are currently purchased from Boostec for further testing.
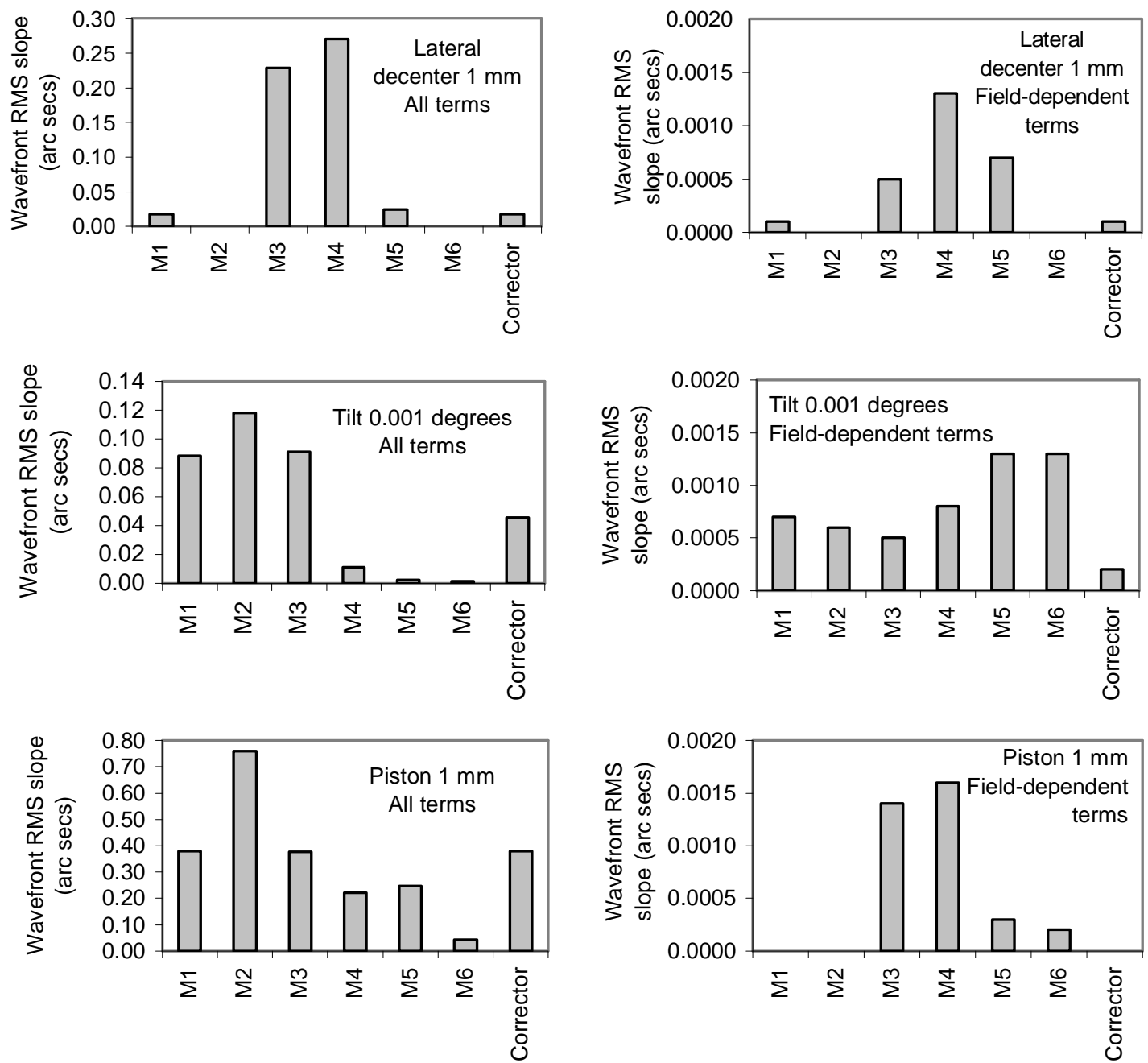

Fig. 3. Sensitivity to decenters

Substantial effort is also being put on the characterization of the optical properties of the telescope, with emphasis on the effect of segmentation on the Point Spread Function, particular attention being paid to imaging of exoplanets. A comprehensive review of diffraction artifacts resulting from segmentation errors, segments edge misfigure and intersegment gaps is provided by Yaitskova et $\mathrm{al}^{22}$. It appears that such artifacts could have brightness of the order of $10^{-4}$ to $10^{-5}$ of the peak of the PSF, pupil rotation not being taken into account. It appears advisable that imaging of faint companions or exoplanets be made under conditions of non-negligible pupil rotation, so as to disentangle diffraction artifacts -be they associated to segmentation or segment failure- from the science target.

\section{TELESCOPE STRUCTURE AND KINEMATICS}

The current layout of the telescope structure and facilities is shown in Fig. 4. To the left is the sliding enclosure, partially open. To the right, the maintenance building, which also serves as support for the four primary mirror covers once they are moved out of the telescope. The telescope itself rotates on azimuth rings, some of which clearly visible in Fig. 4. Up-to-date descriptions of the telescope structure and its properties are presented elsewhere ${ }^{11,12}$. 
Several design directions have been explored, including decoupled structures, in parallel to optical designs. Designs inspired from radio telescope ones had to be dropped, the much longer focal ratio of optical primaries not allowing such designs to provide reasonable stiffness. In parallel to the optical design progressing from a four-mirror solution with 136-m primary-secondary mirror separation and 4 arc minute field of view ${ }^{1}$, to a six-mirror one with $95-\mathrm{m}$ separation and 10 arc minute field of view ${ }^{20}$, the structure evolved towards a compact and stiff construction made out

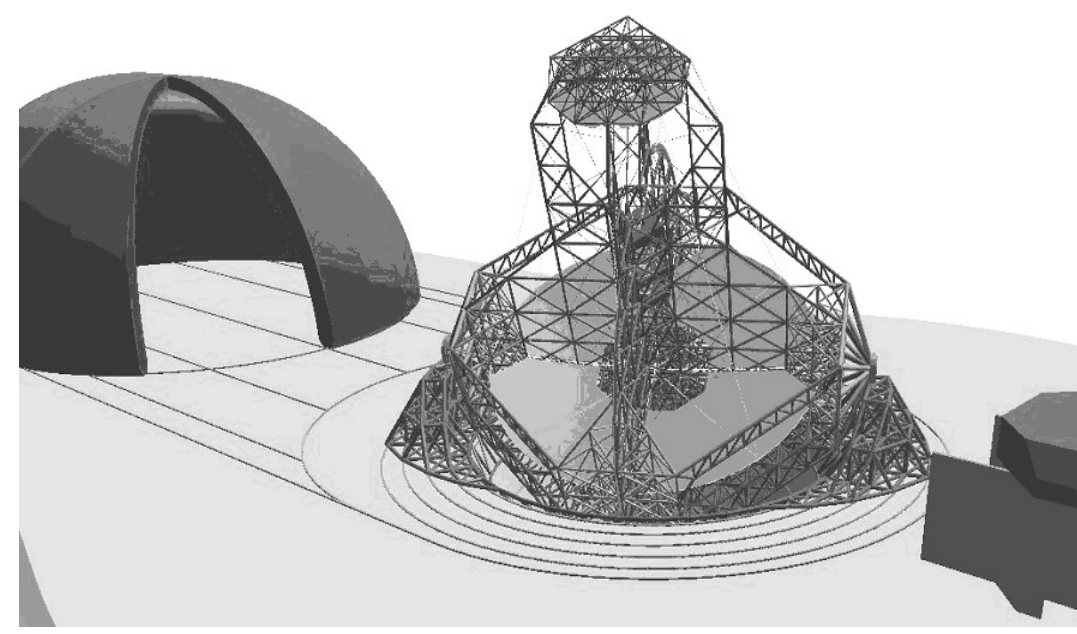

Fig. 4. Layout of the telescope structure and facilities. of nearly all-identical steel modules. In the process, the moving mass decreased from an initial 45,000 down to 13,100 tons, while the first locked rotor frequency increased from 0.5 to $2.1 \mathrm{~Hz}$. Moderately lightweight Silicon Carbide segments for the primary and secondary mirrors would bring the rotating mass down to 8,500 tons and the first eigenfrequency up to $2.3 \mathrm{~Hz}$. Wind flow simulations confirm that the first eigenfrequency is within safe limits with respect to wind excitation. It should be noted that the telescope is de facto extremely lightweight in relation to its size - scaling up traditional designs to OWL dimensions would easily lead to half a million tons, i.e. a 40 times increase.

Static decenters of the primary and secondary mirrors under gravity loads are given in table 3 . Decenters of the corrector are about $1 / 6^{\text {th }}$ that of the secondary mirror. As will be shown later, those can easily be taken care of by presetting and active optics. Even though the telescope is operating in open air, the wind-generated decenters are quite modest-and pretty innocuous in view of the low sensitivity of the design towards decenters. The adverse effect of an enclosure appears clearly from simulation runs. According to these, quasi-static errors (up to $0.5 \mathrm{~Hz}$ ) could effectively be reduced by a factor 2 by the shielding effect of the maintenance building, when the latter is located upwind from the telescope. Conversely, dynamic errors (frequencies above $0.5 \mathrm{~Hz}$ ), which are more preoccupying, tend to increase by a factor 2 to 3 . As it would be practically impossible to perfectly shield a telescope even a fraction the size of OWL from wind excitation, we argue that the only way out is suitable wavefront control. In this context, decreasing quasistatic errors at the cost of increased dynamic effects - pressure turbulence generated by the enclosure's discontinuitiesonly increases the complexity of critical control systems. Enclosures also generate hardly predictable thermal turbulence, which are likely to fall outside the spatial frequency range of adaptive optics and to irremediably hinder the performance of the telescope. The fact that thermal control is already recognized as a tricky and expensive issue in much smaller seeing-limited telescopes should be taken as a dire warning. In short, a co-rotating enclosure is not only an expensive cost position; it is also adversely affecting performance and is a source of unpredictable risks, with potentially dramatic

\begin{tabular}{|l|c|c|c|}
\hline Mirror & $\begin{array}{l}\text { Piston (uz) } \\
{[\mathrm{mm}]}\end{array}$ & $\begin{array}{l}\text { Tilt (rotx) } \\
{[\operatorname{arcsec}]}\end{array}$ & $\begin{array}{l}\text { Decenter (uy) } \\
{[\mathrm{mm}]}\end{array}$ \\
\hline M1 & -4.3 & -48 & 1.1 \\
M2 & -8.9 & 45 & -31.9 \\
\hline M2 - M1 & -4.6 & 93 & -33.0 \\
\hline
\end{tabular}

Table 3. Mean displacements under differential gravity load $\left(\mathrm{z} \sim 0^{\circ}-60^{\circ}\right)$. consequences.

Fig. 5 shows the distribution of wind speed and total pressure derived from a wind flow simulation, with a wind speed of $10 \mathrm{~m} / \mathrm{s}$ and the telescope pointing $20^{\circ}$ from zenith. Other load cases are currently being simulated and preliminary results are presented elsewhere ${ }^{12}$. Besides velocity and pressure distributions, the simulations provide PSD of wind pressure on 500 sample points distributed on the structure and optical surfaces. These can be subsequently input to FE models. 


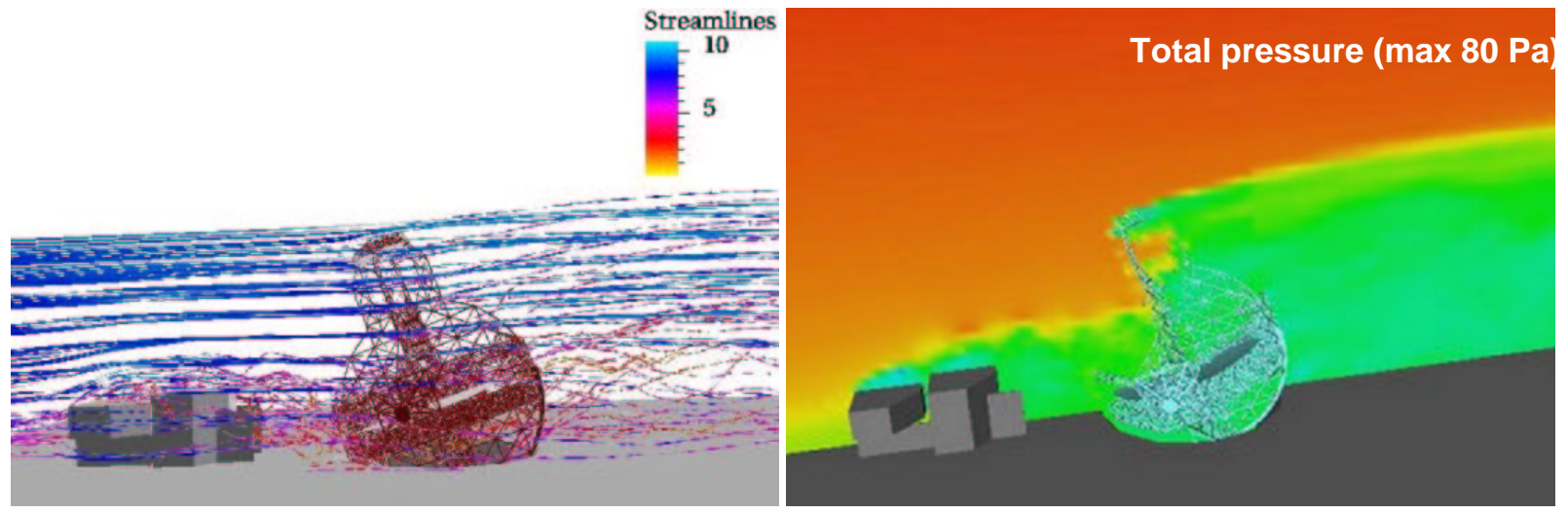

Fig. 5. Wind velocity and pressure distribution; free wind speed $10 \mathrm{~m} / \mathrm{s}$.

Industrial studies are under way to identify optimal fabrication, assembling and maintenance solutions for the nodes and beams of the structure's modules. So far all results are in excellent agreement with initial performance, cost and schedule estimates.

A conceptual design of the corrector structure is shown in Fig. 6. Actuators provide the necessary degrees of freedom for coarse centering and refocusing. For maintenance the corrector is extracted from the telescope by a translation towards M2, telescope horizontal. Interfaces allow separating the groups M3-M4 and M5-M6.

The telescope kinematics has been the subject of particular attention. The initial baseline consisting of hydraulic pads and direct drives has been replaced by a friction drive solution. A comprehensive description of the concept is presented elsewhere ${ }^{11}$. In brief, friction drives provide a solution with higher performance, allow

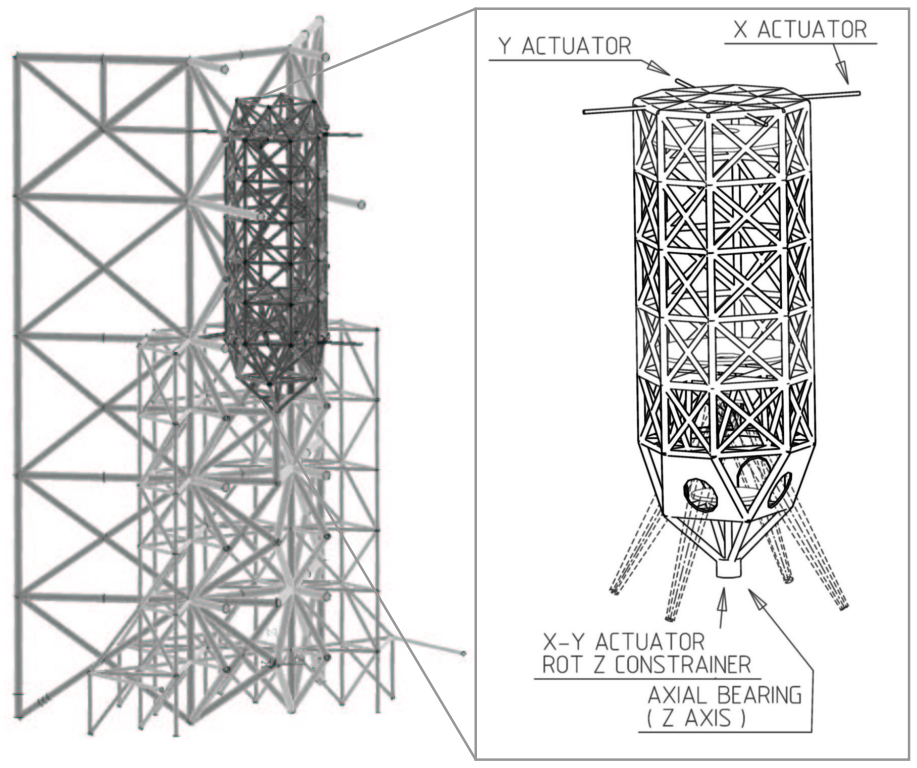

Fig. 6. Conceptual design of the corrector structure. The left picture shows the corrector inside the telescope inner structure. a much better transfer and distribution of loads, and relax fabrication tolerances at an appreciably lower cost. In this concept the kinematics is provided by a large number of bogies allowing a proper distribution of loads onto the tracks.
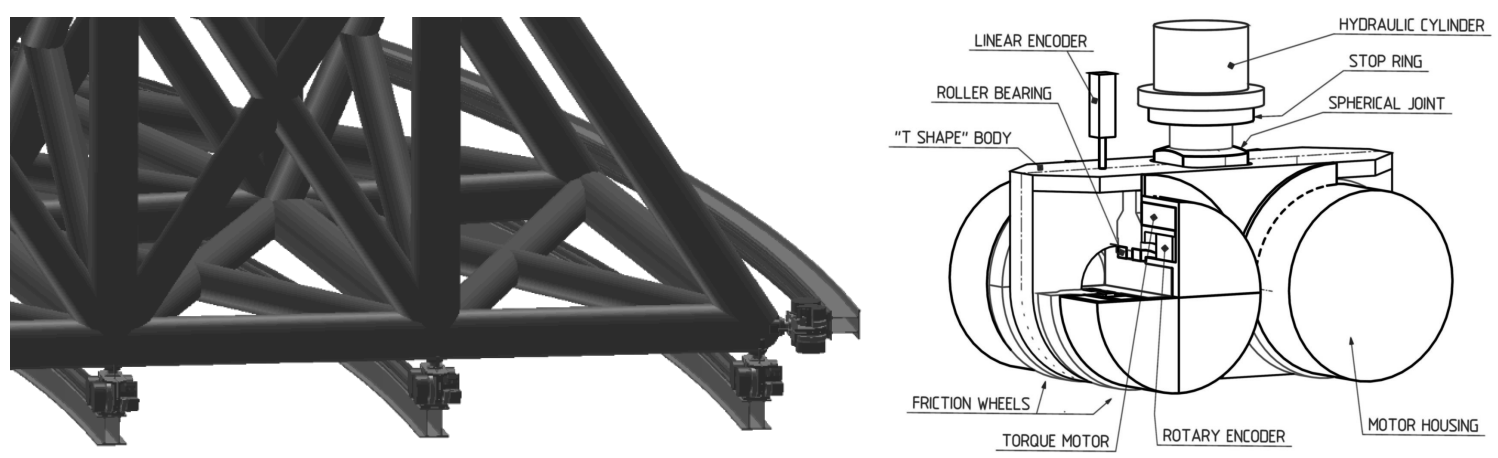

Fig. 7. Friction drives. Left: as mounted on azimuth kinematics. Rails would be embedded into the concrete foundation. Right: layout of a single bogie. 
Merging drive and bearing functions into friction-type bogies (Fig. 7) reduces the complexity and cost of the design and allows constraining the telescope in hyper-static mode. The large number of bogies, about 250 on the azimuth ring and 60 on the altitude structure, ensures a smooth and homogeneous transfer of the loads to the site soil. Each bogie has 4 spherical wheels for the azimuth and altitude (cradle) flat tracks. Each wheel, with a diameter of $630 \mathrm{~mm}$, is independently driven by commercially available brushless ring torque motor equipped with an angular encoder. The required mean angular accuracy is 3 arc minute.at the wheel-motor axis.

The drives are dimensioned to allow a blind angle at zenith of $\pm 0.5^{\circ}$, a maximum acceleration of $0.1 \% \mathrm{~s}^{2}$ and velocity of $0.5 \%$, under conservative assumptions for friction and wind-induced torque. These figures are identical to those of the VLT.

\section{NON-ADAPTIVE WAVEFRONT CONTROL}

While from a physical point of view, adaptive and active optics may be seen as realizing the same function, the corresponding error sources have substantially different amplitudes and time spectra, which translate into different correction and metrology requirements. It is quite plausible that one day technology will allow these to be addressed globally, and the progress of the LBT team in designing and producing a 1-m class adaptive secondary mirror ${ }^{9}$, with impressive amplitude and bandwidth correction capabilities, may arguably indicate that this day is not as remote as it may seem. Within the time frame of OWL and in view of its size, however, it appears reasonably cautious to assume that technology will only allow partial overlap between adaptive and active optics.

We therefore require residual telescope errors to remain within a fraction of the adaptive optics correction capability, not the major part of it. This implies the serial or parallel closing of several wavefront control loops, with decreasing correction amplitudes and increasing time frequencies. We distinguish four such loops: pre-setting, field stabilization, active optics, phasing, each associated to specific metrology and subsystems (Fig. 8).

The purpose of pre-setting is to ensure that the system is in such state that subsequent loops will allow it to rapidly converge towards a compliant one. It shall, in particular, ensure reasonable pointing and collimation accuracy, and wavefront quality within the range of active optics (conservatively one arc second or less). It relies on internal metrology (e.g. autocollimators and/or laser metrology) and look-up tables for input. Most of the errors being associated to gravity and temperature, the corrections are quasi-static. Table 4 outlines preliminary characteristics of pre-setting, assuming a most complex scheme i.e., one that does not rely on existing redundancy between some of the degrees of freedom. The accuracy requirements correspond to $\sim 2.7$ and $\sim 0.6$ arc seconds residuals in pointing and image rms size, respectively. The issue is not so much image quality -VLT experience shows that the active optics loop could be closed with initial image quality worse than predicted here- but pointing. Relaxed tolerances could be accommodated, however at the probable cost of minor additional overheads.

\begin{tabular}{|c|c|c|c|}
\hline Degree of freedom & Range & Typical accuracy & Input \\
\hline Corrector rotation about M2 vertex & 200 arc seconds & 3 arc seconds & Look-up tables, autocollimators \\
\hline Refocus & $25 \mathrm{~mm}$ & $1 \mathrm{~mm}$ & Look-up tables, laser metrology \\
\hline lateral decenters & Expected $<5 \mathrm{~mm}$ & $1 \mathrm{~mm}$ & Look-up tables, laser metrology \\
\hline tip-tilt & Expected $<30$ arc seconds & 4 arc seconds & Look-up tables, autocollimators \\
\hline active deformation & Expected $<50 \mu \mathrm{m}$ & $10 \mu \mathrm{m}$ RMS & Look-up tables, force sensors \\
\hline lateral decenters & Expected $<5 \mathrm{~mm}$ & $1 \mathrm{~mm}$ & Look-up tables, laser metrology \\
\hline tip-tilt & Expected $<30$ arc seconds & 4 arc seconds & Look-up tables, autocollimators \\
\hline active deformation & Expected $<50 \mu \mathrm{m}$ & $10 \mu \mathrm{m}$ RMS & Look-up tables, force sensors \\
\hline refocus & Expected $<5 \mathrm{~mm}$ & $1 \mathrm{~mm}$ & Look-up tables, laser metrology \\
\hline tip-tilt & Expected $<30$ arc seconds & 5 arc seconds & Look-up tables, autocollimators \\
\hline lateral decenters & Expected $<5 \mathrm{~mm}$ & $1 \mathrm{~mm}$ & Look-up tables, laser metrology \\
\hline tip-tilt & Expected $<20$ arc seconds & 5 arc seconds & Look-up tables, autocollimators \\
\hline
\end{tabular}

Table 4. Outline of possible pre-setting characteristics.

Field stabilization is to be provided by M6 unit, which is located in the exit pupil. The sensitivity is 0.16 arc second on-sky depointing per arc second of mirror tilt. Wind flow simulations indicate that under conservative assumptions the maximum expected frequency is $\sim 5 \mathrm{~Hz}$, most of the disturbance occurring below $1 \mathrm{~Hz}$. It is yet unclear whether the correction can be taken over by the adaptive M6 mirror or whether the whole unit should be fitted in a tip-tilt mount. Further simulations for the effect of wind on one side, and evaluation of large adaptive mirrors technologies on 
the other one, are required to draw conclusions. For the time being we assume that a tip-tilt mount will be required, possibly with limited bandwidth and accuracy, residuals being taken care of by the adaptive mirror.

The third loop is active optics, including focus, decentering coma correction, and active deformation of M3 and M4.

Several degrees of freedom allow to compensate decentering coma without introducing depointing (e.g. rotation of any of the M3, M4 or M5 mirrors about their center of curvature, or rotations of groups of surfaces about suitable nodes). A rotation of M5 about its center of curvature is plausibly the simplest approach, with a sensitivity of $0.065 \mu \mathrm{m}$ Zernike coma coefficient per arc second of rotation. Active re-focusing is most suitably performed by axial translation of the mirror M5, with a sensitivity of $20 \mu \mathrm{m}$ wavefront defocus per mm of mirror translation. A typical wind flow simulation shows that in open air, with $10 \mathrm{~m} / \mathrm{s}$ wind speed, the defocus associated to M1 and M2 piston should remain within 1-2 $\mu \mathrm{m}$ wavefront in the band [0-1] Hz, and completely negligible beyond.

Other modes have to be taken care of by actively deforming M3 and M4. As previously explained ${ }^{18}$, bi-conjugate active optics in OWL requires several guide stars (three as a minimum) but those can be much fainter than with the VLT, and it allows for a more extended control of the telescope prescription. It is yet to be assessed whether low spatial frequency deformations of the primary and secondary mirrors under wind buffeting would be better compensated by the segment supports only, or whether part of the correction should be off-loaded onto the quaternary mirror. Were this to be the case, the active optics wavefront sensor scheme would have to follow the same principles as those applicable to layer-oriented multi-conjugate adaptive optics (MCAO) to isolate the in-pupil disturbances, however with much lower sampling and longer integration times. The M4 cell and force actuators would have to be dimensioned for sufficient bandwidth. According to early estimates, wind-induced M1 astigmatic-like deformations are less than $\sim 0.5 \mu \mathrm{m}$ RMS wavefront at frequencies beyond $5 \mathrm{~Hz}$. This is, however, the result of an overly conservative assessment and we expect a reduction of bandwidth and/or amplitude. The VLT primary mirror support system was designed to allow a $1 \mathrm{~Hz}$ bandwidth for fear of wind, which never truly materialized.

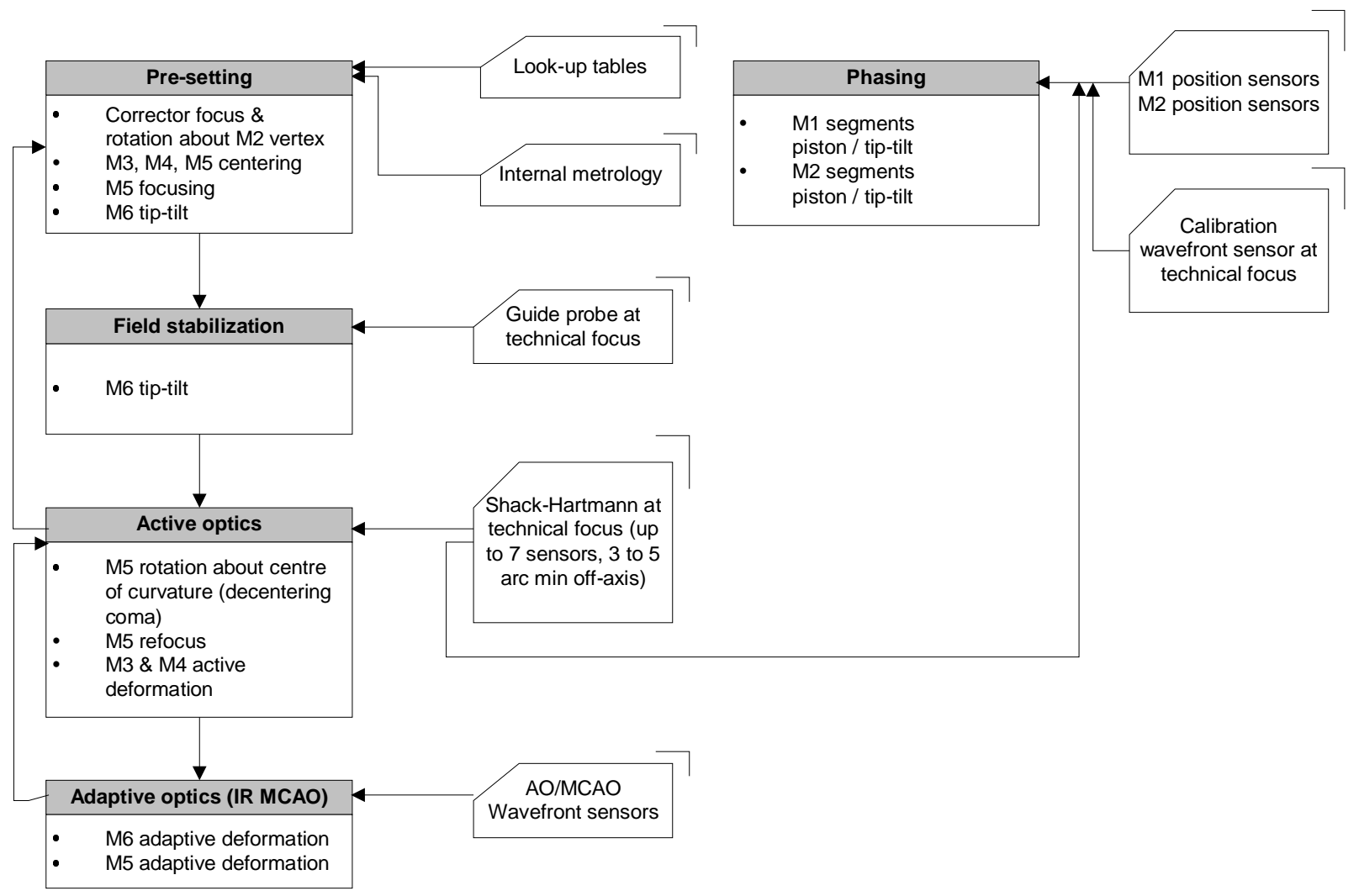

Fig. 8. Outline of the wavefront control loops. 
While an overlap between active deformation of the quaternary mirror and phasing of the segmented mirrors may relax phasing requirements on low order modes, a distinct phasing loop is in any case required. The baseline solution for OWL relies on the same approach as in the Keck. Position sensors conveniently located at the back or the edges of the segments provide, in real time, measurements of the inter-segments steps, down to a few nanometers accuracy. Whichever technology such sensors rely on, periodic calibration of their readings appears necessary. This calibration is ideally performed on-sky; Chanan et $\mathrm{al}^{23}$ have successfully developed a wavefront sensing technique which allows re-calibration of the Keck sensors, within adequate accuracy and at an affordable cost in terms of operational overheads -typically a few hours on a monthly basis. According to Chanan the technique is scalable to a very large number of segments, within existing technology. In the case of OWL, which has segmented primary and secondary mirrors, the technique would most likely require two wavefront sensors, each fitted with proper pupil masks centered on the images of segment boundaries. Those would provide independent calibrations of each segmented mirror. In contrast with the Keck, the relatively poor sensitivity of position sensors for low order modes is mitigated by the fact that these modes are sensed and corrected in real time by the active optics loop.

Alternative calibration techniques are being explored within the framework of a European Community-funded Research and Training Network (RTN) on adaptive optics for extremely Large Telescopes. Those include an evolution of the Chanan technique, due for implementation in the Spanish GTC 10-m telescope ${ }^{24}$, curvature-based methods ${ }^{25,26}$, pyramid wavefront sensors ${ }^{27}$, and Mach-Zehnder interferometry ${ }^{16}$. The latter is jointly explored by the Laboratoire d'Astrophysique de Marseille and ESO. All these alternatives allow rather faint (V 13 to 16 and possibly beyond) reference sources, thereby allowing the calibration to be executed more frequently than in the Keck, using off-axis references and without loss of science time. With some variations from one method to the other, neither accuracy nor capture range seem to pose insurmountable problems. The added difficulty of individually phasing two surfaces favours methods that retrieve the phase information from pupil images, namely curvature, pyramid, and MachZehnder wavefront sensors. In the latter case, it has been shown ${ }^{16}$ that simple Fourier filtering of the data allows disentangling of the phasing information associated to each segmented surface. An example is shown in Fig. 9 with a reduced number of segments; using OWL aperture would not allow suitable printed reproduction. To varying extent all calibration techniques are fairly insensitive to atmospheric turbulence. As will be explained later, ESO's plan is to test the most promising candidates on the sky.

It should be mentioned that occasional phasing failures, be they associated to obscuration forbidding the calibration of a few segments or to actuators or sensors malfunctions, have negligible impact on the performance of the telescope. Not only does the collecting power of a single segment represent a negligible fraction of that of the total aperture, but in addition its individual PSF is spread over a much larger area. It can also be shown that the PSF of failing segments could be defocused by use of the hydraulic maintenance jacks normally devoted to their handling, thereby blurring the ghost images they would otherwise deliver.

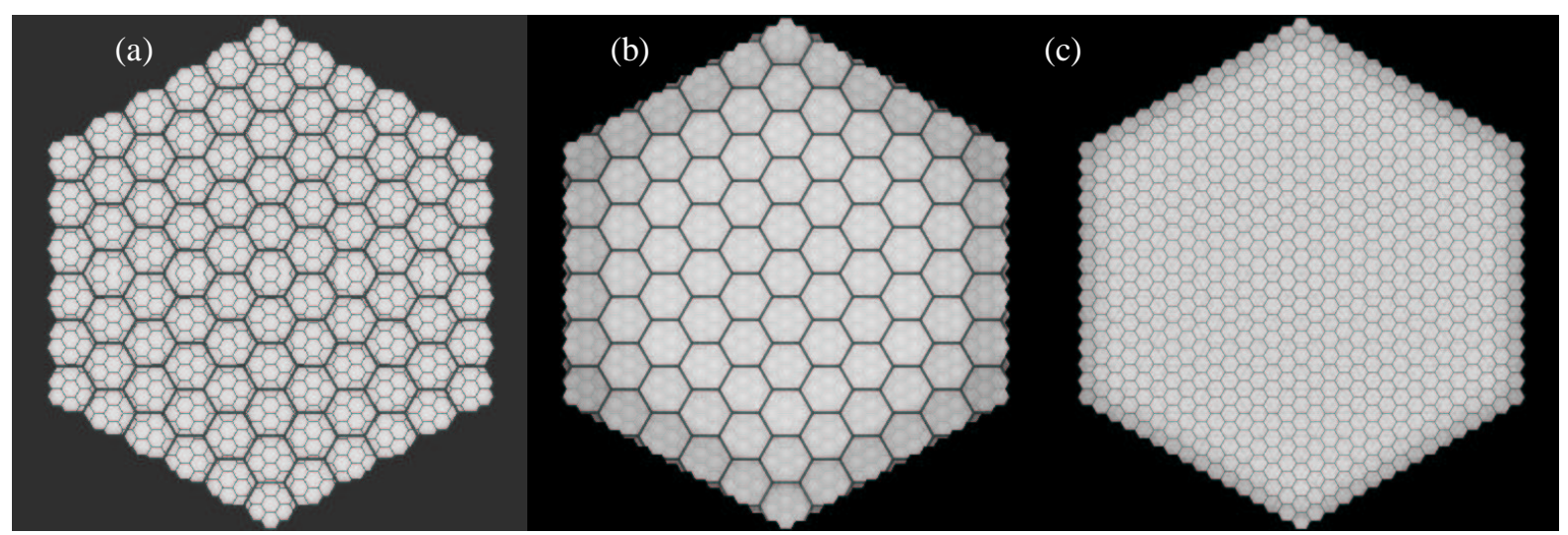

Fig.9. Simulated Mach-Zehnder white light images of an aperture with (a) two superimposed segmentation patterns and $(\mathrm{b}, \mathrm{c})$ after Fourier filtering. See also Montoya et $\mathrm{al}^{16}$, this conference.

Preliminary figures for the expected maximum frequency of each wavefront control loop are given in table 5. These figures are very tentative and subject to changes upon completion of further simulations. For seeing-limited operation, 
requirements could be substantially relaxed as disturbances at the upper limit of the bandwidth are expected to be small in wavefront slope, most of the corresponding modes being of low spatial frequency.

\begin{tabular}{|l|l|}
\hline Wavefront control loop & Maximum frequency (tentative) \\
\hline Pre-setting & $\leq 0.01 \mathrm{~Hz}$ \\
Field stabilization & $\leq 5 \mathrm{~Hz}$ \\
Active optics & \\
Active coma correction & $\leq 1 \mathrm{~Hz}$ \\
Active focusing & $\leq 1 \mathrm{~Hz}$, optionally $\leq 5 \mathrm{~Hz}$ \\
Active deformation M4 & $\leq 2 \mathrm{~Hz}$, optionally $\leq 5 \mathrm{~Hz}$ \\
Active deformation M3 & $\leq 0.1 \mathrm{~Hz}$ \\
Phasing & $\leq 0.1$ in stand-by, $\leq 5 \mathrm{~Hz}$ in operation \\
On position sensors & A few per night at most \\
\hline
\end{tabular}

Table 5. Expected maximum frequency of each wavefront control loop.

\section{ADAPTIVE OPTICS}

Adaptive Optics (AO) capability is a mandatory -and the most demanding- requirement for OWL. Several possible modes must be implemented to comply with the complete palette of science requirements ${ }^{28}$. Those are, in brief:

- $\quad$ wide-field (up to 6 arc minutes) seeing-reduction to less than $\sim 0.2$ arc seconds.

- classical or single conjugate, narrow-field adaptive optics (SCAO); the field of view is set by the isoplanatic patch.

- multi-conjugate adaptive optics (MCAO), with a field of view of up to 30 arc seconds in the visible and 3 arc minutes in the infrared.

- Extreme adaptive optics XAO / XMCAO, allowing relatively small field (1-10 arc seconds) but requiring high Strehl Ratio (up to $80 \%$ in K, $30 \%$ in the visible)

These capabilities will be integrated gradually, the three first ones being planned to start operation while the telescope aperture is being filled (see section 8 of this article).

The roadmap towards OWL adaptive optics relies on the convergence of several paths, some related to concepts, others to technology. Concepts, and to some extent technology, can be explored by means of simulations, models, and eventually, experiments and prototypes. We will outline our approach towards modeling and simulations first, and present our plans for technology development second.

Current software is not adequate to simulate OWL (MC)AO system(s), because it is mostly written in high-level (Matlab or IDL) language, which are not designed to run in a cluster. In order to overcome the limitations of a single pc, we also plan to run a new simulation software in parallel on a cluster of 20-30 Linux-PCs, with a total of $~ 40$ Gbytes of RAM and $40 \mathrm{GHz}$ of cluster CPU, which will allow full AO simulations of OWL.

Two applications are being ported to this HW/SW architecture: an analytic (covariance) code and a full numerical one. The first will allow to explore rapidly a large parameter space (in the open loop case) and to roughly set key parameters. The second will include almost all AO system details in a limited number of cases, in closed loop. It should be noted that both LGS and NGS cases can and will be simulated. Both analytical and numerical code conversions are well underway. The parallel analytical code has been used to compute the shape of the $100,000^{\text {th }}$ Karhunen-Loeve polynomial (6 PCs being used). A very preliminary version of the numerical code (only parts of it have been ported from IDL to $\mathrm{C}$ for the moment) has been shown to be able to simulate the AO system (single guide star and Deformable Mirror DM) of a 30m telescope. Details about the simulation effort at ESO and very preliminary results are presented elesewhere ${ }^{13,14}$. It is foreseen that the analytical code will provide the first results on a $100 \mathrm{~m}$ telescope AO system by mid 2003. As for the numerical code, it is expected to simulate a 30-50m ELT within the next year (2003). For a full numerical OWL simulation, access to a supercomputer will probably be necessary, which should happen within the next 2-5 years. However, the analytical software results and extrapolations from the 30-50m case should provide a wealth of information about the OWL AO system before that date. 

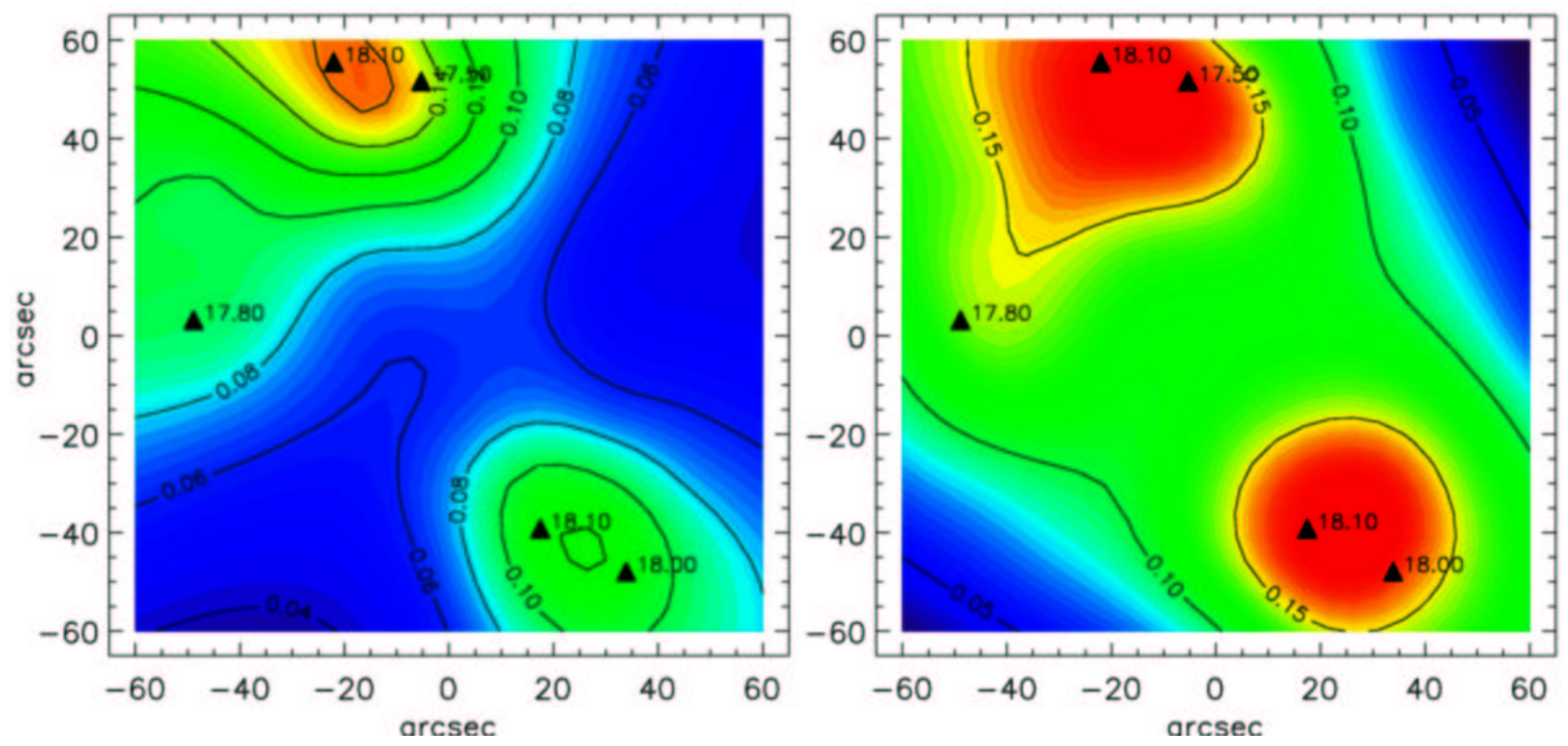

Fig. 10. Predicted Strehl maps at the South galactic pole. Left: 8-m aperture; right: OWL. See also Fedrigo et al ${ }^{14}$.

It should not be concluded, however, that no simulation is possible yet. Indeed, Fedrigo et al $^{14}$, by making simplifying assumptions, have recently succeeded in modeling single- and multiple-field AO and in predicting Strehl maps for OWL. These simulations use realistic NGS asterisms and have been validated by applying them to VLT and comparing the results with those provided by detailed, more complete simulations. The benefit of the large aperture in terms of Strehl distribution across the field appears clearly from the results. Fig. 10 shows the predicted Strehl maps in $\mathrm{K}$ obtained in a 2 arc minutes field centered on the South galactic pole, with dual conjugates, layer-oriented AO and 5 reference stars of magnitude $17.9 \pm 0.3$ within the $2 \times 2$ arc minute ${ }^{2}$ field. The left side picture corresponds to an 8 -m aperture and the right one to OWL. Triangles correspond to the location of the reference stars and are labeled with the star's magnitude. The adaptive correction capability of the 8-m aperture corresponds to that of ESO's Multi-conjugate Adaptive optics Demonstrator (MAD, see section 7 of this article), with dual conjugates. Sampling of the ground layer is $8 \times 8$, sampling of the $8-\mathrm{km}$ one is $7 \times 7$. For OWL, sampling of the ground layer is $100 \times 100$ and of the 8 -km layer is $53 \times 53$. The simulations used to produce Fig. 10 also allow determining sky coverage with NGS and evaluating performance as a function of reference star brightness. Sky coverage for typical Strehl of $20-40 \%$ is about $90 \%$ at $20^{\circ}$ off the galactic plane, down to $\sim 15 \%$ at the galactic poles.

A parameter that has marginal influence with 8-m class telescopes but becomes crucial with OWL is the outer scale of atmospheric turbulence. The effects of the outer scale $\mathrm{L}_{0}$ were first put in evidence in interferometr $y^{29,30}$. In particular, it has been shown that the variance of the Optical Path Difference (OPD) due to atmospheric turbulence does not increase as the power of the baseline (as expected with an infinite $\mathrm{L}_{0}$ model) but becomes independent on the baseline ${ }^{31}$ after few tens of meters. A finite $\mathrm{L}_{0}$ is also expected to impact the performances of a $100-\mathrm{m}$ diameter telescope, even when no adaptive optics (AO) correction is applied to phase corrugation.

With an infinite $\mathrm{L}_{0}$, the Full Width at Half Maximum (FWHM) of the long-exposure Point Spread Function (PSF) of an arbitrary large telescope is FWHM $=0.98 \lambda / r_{0}$, where $r_{0}$ is the atmospheric coherence length. However, with a finite $\mathrm{L}_{0}$ the PSF is strongly dependent on the modal spectrum of the phase fluctuations. And it has been shown that the modal distribution of the phase energy changes when $\mathrm{L}_{0}$ becomes smaller than the telescope ${ }^{13}$. 
Fig. 11 shows the Strehl ratio (SR) and FWHM as a function of $\mathrm{L}_{0}$ in $\mathrm{V}(0.55 \mu \mathrm{m}), \mathrm{J}(1.25 \mu \mathrm{m})$ and $\mathrm{K}(2.2 \mu \mathrm{m})$ bands with $\mathrm{r}_{0}=15 \mathrm{~cm}$ in $\mathrm{V}$. The FWHM is given in units of $\lambda / \mathrm{D}$, D being the telescope diameter. FWHM=1 corresponds to diffraction limited angular resolution. The SR and FWHM plotted here are derived from the combined telescopeatmosphere PSF when no AO correction is applied. Straight lines on the right graph gives the FWHM of seeing limited PSF. For $\mathrm{J}$ and $\mathrm{K}$ bands, near-diffraction limited images are obtained when $\mathrm{L}_{0}$ is much smaller than $\mathrm{D}$, typically, below $15 \mathrm{~m}$ in $\mathrm{K}$ and below $7 \mathrm{~m}$ in $\mathrm{J}$. However, the dynamic range of the images is poor with $\mathrm{SR} \sim 10 \%$ in $\mathrm{K}$ and $\sim 0.1 \%$ in $\mathrm{J}$ for the smallest $\mathrm{L}_{0}(\sim 5 \mathrm{~m})$ and decreasing rapidly to $\sim 0.1 \%$ in $\mathrm{K}$ and $>0.01 \%$ in $\mathrm{J}$ for a $\mathrm{L}_{0}$ of $15 \mathrm{~m}$.
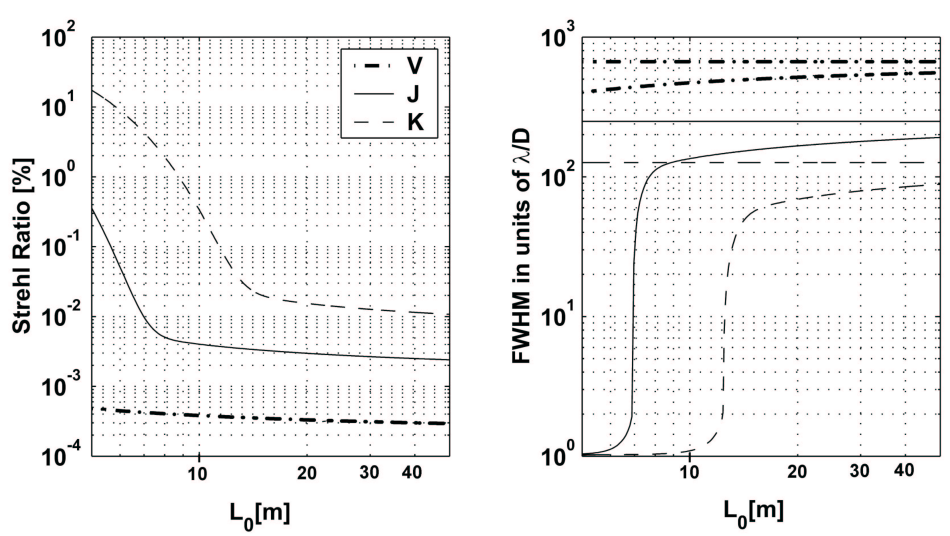

Fig. 11. OWL Strehl Ratio and FWHM of the long-exposure, uncorrected PSF as a function of outer scale $\mathrm{L}_{0}$ in $\mathrm{V}, \mathrm{J}$ and $\mathrm{K}$ bands.
These results show that, in the case of high signal-to-noise ratio and favorable seeing conditions, very high image resolution can theoretically be achieved without any adaptive optics correction. In order to quantify the occurrence of favorable seeing condition for a 100-m diameter telescope and taking Paranal as a test case, we used data measurement realized by the Generalized Seeing Monitor in December $1999^{32}$. Using a total of 1884 measurements recorded during 19 nights, simultaneous values of $\mathrm{L}_{0} \leq 15-\mathrm{m}$ and $\mathrm{r}_{0} \geq 15 \mathrm{~cm}$ have been observed $8.3 \%$ of the time. It is doubtful that this effect could translate into a useful observation mode, as image stabilization to diffraction-limited accuracy would be required to compensate for vibrations and tracking errors.

These simulations serve the dual purpose of predicting performance and optimising control parameters on one side (with surprises as those produced by Conan et $a l^{13}$ and Fedrigo et $a l^{14}$ as spectacular by-products), and assessing technological requirements, be they related to active components or to metrology, on the other one. A proper modelling of atmospheric turbulence, in particular, is of utmost importance to set the necessary dynamic range, spatial and time frequencies of adaptive corrections.

In the current design, mirrors M6 and M5, conjugated to 0 and $8 \mathrm{~km}$, respectively, are earmarked for near-IR MCAO. The adaptive, 2.35-m M6 would be integrated first, along with a temporary, non-adaptive M5 unit. This will allow wide-field seeing reduction and classical adaptive optics to be operated at an early stage, starting with a 45-m aperture, down to $\mathrm{r}_{0} \sim 1-\mathrm{m}$ (assuming the same technology as in the $\mathrm{LBT}^{9}$, with $28 \mathrm{~mm}$ actuator interspacing). The temporary M5 unit would be replaced about a year later by a 4.0-m adaptive one, allowing either classical adaptive optics down to $\mathrm{r}_{0} \sim 0.6-\mathrm{m}$ or, in conjunction with M6, near-IR MCAO with a 70-m aperture. Both M5 and M6 adaptive units being dimensioned to the full aperture, they will remain in operation once the aperture is completely filled. We currently assume that they will eventually deliver first-stage correction at shorter wavelength and for XAO or XMCAO, high spatial frequencies being thence compensated by a dedicated active optics unit relying on MOEMs technology.

As for large adaptive mirrors, our intention is to proceed in a stepwise manner, an adaptive secondary mirror for one VLT unit telescope being a major intermediate step. Lessons from the LBT will undoubtedly play a major role but alternative technologies will be explored in parallel.

Metrology requirements, while deemed compatible with moderate technology extrapolation for infrared AO, become extremely challenging in the visible. The first issue is to find suitable reference sources, the second to sample them at the required spatial and temporal rates. The size of OWL plays a positive factor in the overlap of beam footprints at high altitude, which for similar field requirements could be inexistent on much smaller telescopes. This allows for sufficient sky coverage with Natural Guide Stars down to a wavelength of $0.8 \mu \mathrm{m}$, up to the galactic poles ${ }^{14}$. Correction at visible wavelengths will nevertheless require Laser Guide Stars, with the added complexity implied by their finite distance and variability. The second issue is essentially related to detector technology and much will depend on its progress. It should be noted, however, that even in the unfortunate case where access to visible wavelengths would take appreciable delays, the capabilities of OWL with (only) dual-conjugate IR AO capability 
would undoubtedly do more than satisfy scientific demand. Simulations also show that OWL IR AO system should deliver diffraction-limited resolution with a few percent of Strehl Ratio in the visible, under favourable seeing conditions. Should pupil size be the constraining issue, first visible or XAO experiments could also use an aperture temporarily clipped, thereby allowing the telescope to remain competitive at all wavelengths. Admittedly not noble arguments, nevertheless pragmatic ones.

\section{VALIDATING EXPERIMENTS}

While the feasibility of OWL is generally not contested, it is sometimes objected that in view of its size, an "intermediate step" is required, in the assumed form of a scaled-down version of OWL, which we'll christen FOWL (First $O W L$ ) for the ease of reference. A simple risk assessment, however, shows that it is possible to fulfill the scope of an "intermediate step" in a much faster and economical manner.

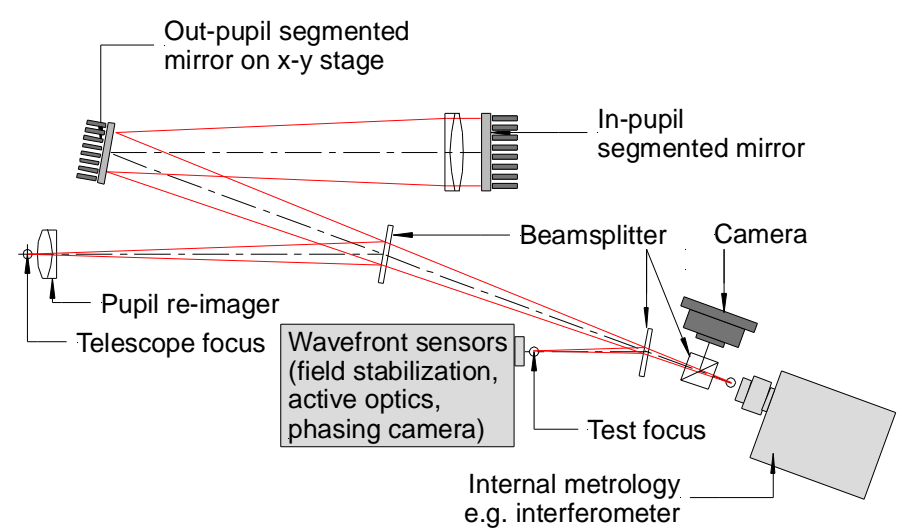

Fig. 12. Schematical layout of the APE test bench.
Risks may materialize through fabrication, integration, or operation. The telescope design relying on proven technologies, fabrication is not a strong incentive for building a FOWL. In virtually all schedule- and cost-sensitive areas, industrial studies and allocations for the ramping up of production lines will do as well as FOWL's construction. Critical components, such as position actuators and sensors, will be subject to competitive designs, and prototypes can be tested under representative conditions for performance and reliability. The situation is somewhat different with integration and operation, where a FOWL could indeed make sense. Integration of the mechanical and optical subsystems is not so much of a concern, as it can be predictably planned and even rehearsed with a limited number of breadboards and mock-up modules. It should also be noted that all functions integrated into OWL have been tested on-sky for years; this was not the case for the first segmented nor the first active telescopes.

The crucial problem will be to successfully integrate all wavefront control functions, ensure that they will have the necessary range, bandwidth and accuracy, and that they can be made reasonably transparent to the user. Range and bandwidth being strongly size- and design-dependent, a FOWL may provide partial information at best, and emphasis should be put, instead, on integration, accuracy and operability. Here again, there are suitable alternatives. If the objective is to test the integration of active optics and segmentation, then the logical way to do it is to turn to the sky an active and segmented telescope. Instead of building from scratch a telescope incorporating these technologies, it is far more convenient to modify an existing one to the same effect. This is the purpose of ESO's Active Phasing Experiment (APE). In this experiment, a fairly simple technical instrument would be mounted onto an active VLT telescope. The instrument would basically consist of a pupil re-imager and a couple of small, segmented mirrors driven by piezoelectric actuators. Its concept is shown in Fig. 12.

One of the segmented mirrors is conjugated to the telescope pupil, the second one, with different segment size and mounted onto an $\mathrm{x}-\mathrm{y}$ stage, emulates OWL secondary mirror, the $x-y$ stage allowing to emulate fielddependent shifts of the segmentation patterns of the two mirrors. Internal metrology, e.g. a two-wavelengths interferometer, permits precise monitoring

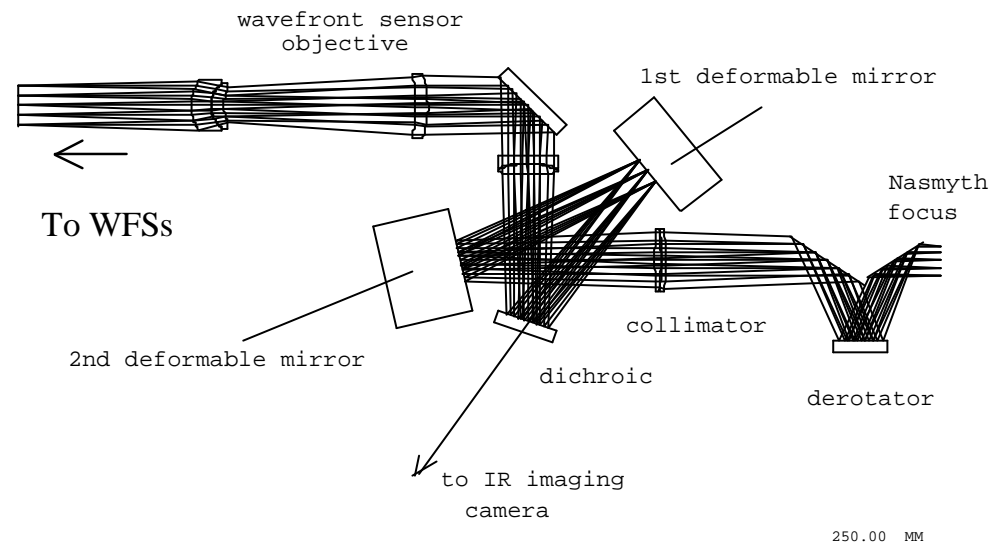

Fig. 13. Optical design layout of the MCAO Demonstrator (MAD). 
of the wavefront errors generated inside the instrument. The wavefront entering the instrument can be monitored and modified at will by the telescope active optics system to simulate expected disturbances in OWL. An imaging or pupil camera can be mounted at one output of the instrument, the other one delivering a test focus. The latter can be used to mount phasing cameras in order to qualify possible phasing techniques, and eventually to mount a combination of wavefront sensors for guiding, active optics and phasing. The combined output of this set of wavefront sensors can be compared to the actual state of the complete system, as delivered by APE internal metrology and the telescope active optics one. OWL wavefront control loops in seeing-limited regime can thereby be experimented and validated. In a second phase, the imaging camera output could be interfaced with the ESO Multi-conjugate Adaptive optics Demonstrator $^{33}$ (MAD, see below) to test the complete phasing-active optics-adaptive optics system.

A MAD APE will not fully replace a FOWL but will permit a comprehensive testing and optimization of metrologies, control systems and software (and, perhaps, reveal the author's fondness for dubious acronyms and incomprehensible statements). A conceptually similar experiment was undertaken by ESO when it decided to implement and test the VLT control system on the 3.5-m New Technology Telescope (NTT). The potential benefit of this experiment could hardly be overstated.

While APE is still in a conceptual design phase, MAD is already under construction, having successfully passed its conceptual design review. Its purpose is to demonstrate Multi-Conjugate Adaptive Optics on-sky, at the focus of a VLT 8-m telescope, by early 2004. A description of the instrument and account of its fabrication status is provided

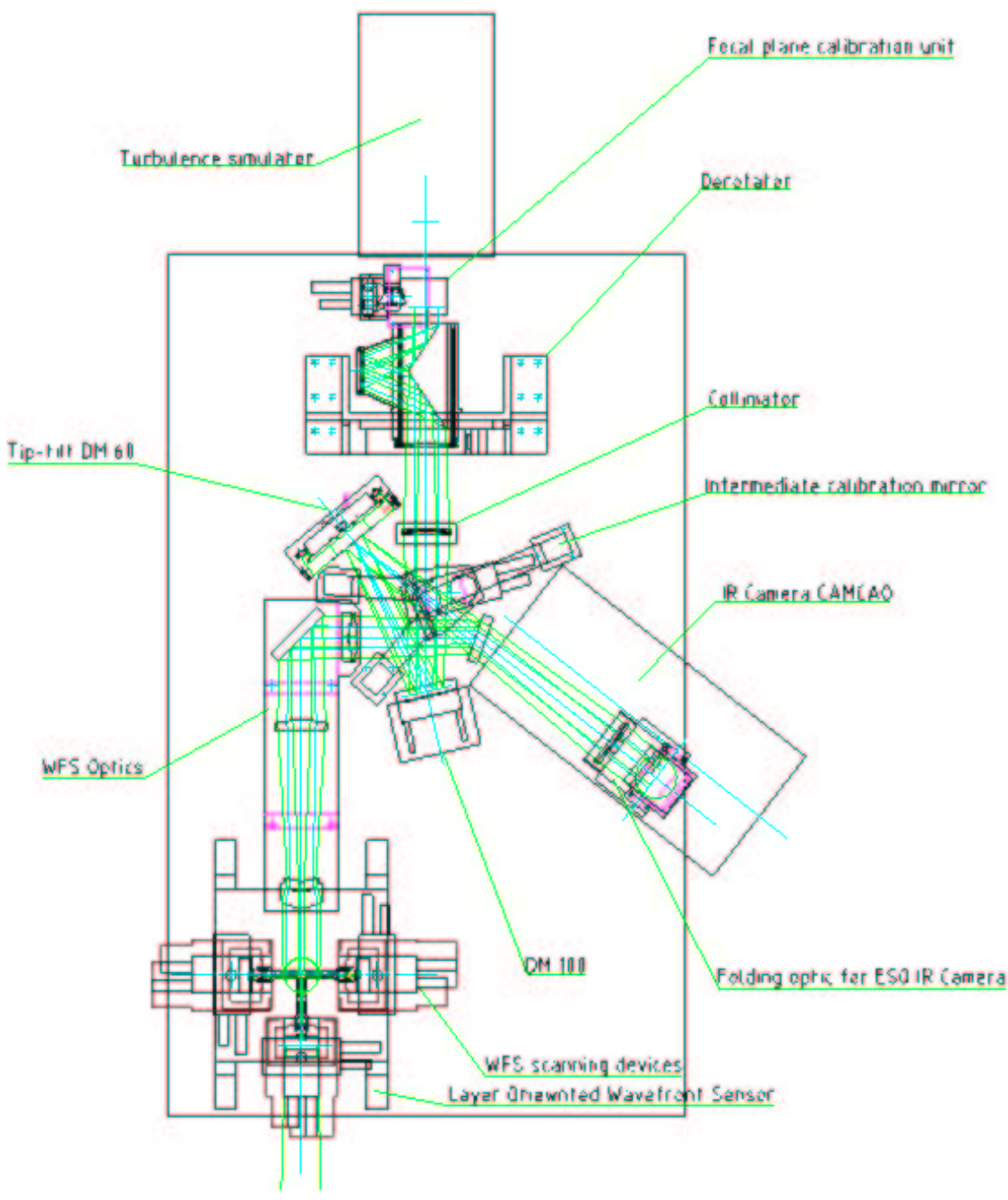

Fig. 14. MAD General Assembly (top side view). See also Marchetti et $\mathrm{al}^{33}$. elsewhere $^{33}$. MAD is a prototype MCAO system performing AO correction over 2 arc minutes on the sky by using bright $\left(\mathrm{m}_{\mathrm{v}}<14\right)$ Natural Guide Stars (NGS). It will assess two different approaches for MCAO correction with two independent wavefront sensing techniques: a Star Oriented MCAO with a Shack-Hartmann wavefront sensor (WFS) sensing simultaneously 3 NGS with 3 Shack-Hartmann WFS, and a Layer Oriented MCAO with a Layer Oriented Wavefront Sensor based on a Multi-Pyramid WFS $^{34,35}$ sensing simultaneously 8 NGS. INAF-Arcetri Observatory supplies the latter. The MAD Real- Time computer architecture is designed in order to support both reconstruction approaches. The layout of the optical design and of the optomechanics are shown in Fig. 13 and 14, respectively. Extensive laboratory testing is planned; hence the turbulence generator included in Fig. 14.

The performance predicted on the basis of extensive simulations is in the range of $30-60 \%$ peak Strehl Ratio, assuming favorable asterisms. Suitable ones have been identified up to the galactic poles. 


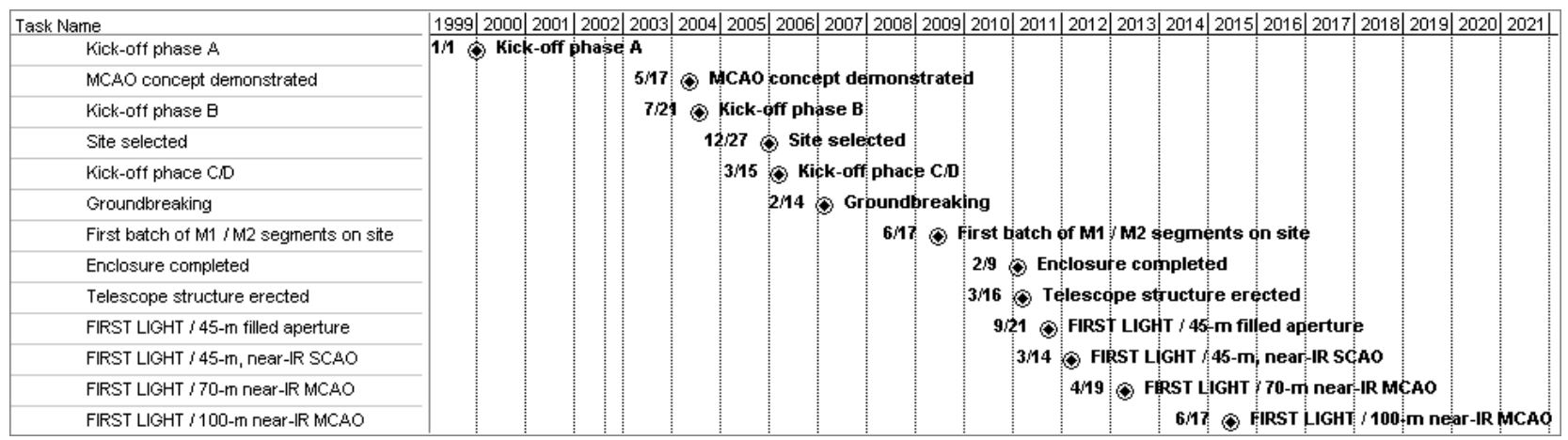

Fig. 15. OWL schedule estimate

\section{SCHEDULE AND COST ESTIMATES}

Current schedules and cost estimates are derived from technical assessments and draft plans, substantial input being taken from industrial studies. The maximum capital investment is constrained to one billion Euros, and the plans should ensure a start of science of operation in the first half of the $2^{\text {nd }}$ decade, full potential being technically possible by 2015 .

The objective of phase $\mathrm{A}$ is to complete conceptual design by 2004 and a validation of the adaptive optics concept through MAD. This phase will be concluded by the delivery of a proposal for the ensuing phases, compliant with the cost and schedule constraints. Would such proposal be approved, a phase B (2004-2008) will follow, which will include competitive preliminary designs of major, time-critical subsystems, as well as prototyping and breadboards of critical components. A full commitment to construction would have to occur early 2006, at the about the same time as final site selection, in order to allow a start of science operations by 2012 and a full completion by 2015. Cash-flow would have to ramp up by 2007 , with the placing of major contracts for the structure and the mirrors.

Of particular importance for OWL schedule is the modular approach, whereby the time-critical telescope components are made of nearly all-identical modules. With the exception of the corrector's mirrors and cells, virtually all telescope components can be shipped in standard containers.

Allowing for a progressive integration of the primary and secondary mirrors, and accepting a start of operation with a reduced potential (45-m in 2012, 70-m by 2013), schedule pressure is transferred from optical to mechanical fabrication and integration. While the former relies on specialized facilities and personnel, the latter does not.

Mechanical fabrication and integration lines can therefore be duplicated at an affordable cost and without substantial increase of investment in fabrication facilities. Once their final design is completed, for example, structural modules

\begin{tabular}{|c|c|c|}
\hline SUMMARY & MEuros & \\
\hline OPTICS & 356 & \\
\hline Primary mirror unit & & 271.4 \\
\hline Secondary mirror unit & & 30.7 \\
\hline M3 unit & & 14.4 \\
\hline M4 unit & & 23.9 \\
\hline M5 unit & & 5.3 \\
\hline M6 unit & & 10.1 \\
\hline ADAPTIVE OPTICS & 100 & \\
\hline MECHANICS & 184 & \\
\hline Azimuth & & 55.4 \\
\hline Elevation & & 25.8 \\
\hline Cable wraps & & 5.0 \\
\hline Azimuth bogies (incl. motors) & & 21.8 \\
\hline Altitude Bogies \& bearings & & 5.5 \\
\hline Mirror shields & & 15.0 \\
\hline Adapters & & 6.0 \\
\hline Erection & & 50.0 \\
\hline CONTROL SYSTEMS & 17 & \\
\hline Telescope Control System & & 5.0 \\
\hline M1 Control System & & 8.0 \\
\hline M2 Control System & & 2.0 \\
\hline Active optics Control System & & 2.0 \\
\hline CIVIL WORKS & 200 & \\
\hline Enclosure & & 40.4 \\
\hline Technical facilities & & 35.0 \\
\hline Site infrastructure & & 25.0 \\
\hline Concrete & & 100.0 \\
\hline INSTRUMENTATION & 45 & \\
\hline Total without contingency & Meuros & 903 \\
\hline Contingency & $11 \%$ & 97 \\
\hline Fixed total & Meuros & 1000 \\
\hline
\end{tabular}

could be procured from several suppliers. The structure is its

Table 6. OWL cost estimate 
own scaffolding, and integration schedule mostly depends on investment in temporary and relatively cheap logistics and personnel. In brief, the combination of standard parts and availability of suppliers allow the structure and kinematics to be produced and assembled within a

time scale comparable to that of a much smaller, less standardized telescope. Fig. 15 shows the current schedule estimate. This estimate already incorporates the results of industrial studies, and is subject to revision as work progresses.

This schedule assumes 1.8-m flat-to-flat M1 segments; blank deliveries would span over 6-7 years after a 3-years phase for the construction of the facilities. All feasibility studies so far conclude that the 6-7 years delivery span could be reduced to 5, with negligible cost increase. This applies equally to "classical" materials (e.g. Zerodur, Astro-Sital, ULE) and lightweight ones (sintered $\mathrm{SiC}$ ). Schedule assumptions for the polishing remain to be crosschecked by industrial studies. It is generally felt that the segments design (all-identical, flat or spherical) should allow a reliable schedule, compatible to that of the blanks.

As for integration, it is assumed that erection of the sliding enclosure and of the telescope structure would not suffer significant interruption as a result of adverse meteorological conditions.

The cost estimate for capital investment is summarized in Table 6. It assumes relatively friendly site properties i.e. moderate altitude (typically 2-3,000-m), access to reasonable infrastructure (e.g. roads, harbor), low seismicity and moderate wind speed (median below $8 \mathrm{~m} / \mathrm{s}$ ).

\section{REFERENCES}

1. R. Gilmozzi, B. Delabre, P. Dierickx, N. Hubin, F. Koch, G. Monnet, M. Quattri, F. Rigaut, R.N. Wilson, The Future of Filled Aperture Telescopes: is a 100m Feasible?; 1998, Advanced Technology Optical/IR Telescopes VI, SPIE 3352, 778

2. A. B. Meinel, An overview of the Technological Possibilities of Future Telescopes, 1978, ESO Conf. Proc. 23, 13.

3. L. D. Barr, Factors Influencing Selection of a Next Generation Telescope Concept, 1979, Proc. SPIE Vol. 172, 8.

4. A. Ardeberg, T. Andersen, B. Lindberg, M. Owner-Petersen, T. Korhonen, P. Søndergård, Breaking the 8m Barrier - One Approach for a 25m Class Optical Telescope, ESO Conf. and Workshop Proc. 42, pp. 75-78, 1992.

5. M. Mountain, What is beyond the current generation of ground-based 8-m to 10-m class telescopes and the VLT-I?, SPIE 2871, pp. 597-606, 1996.

6. P. Dierickx, 1992, J. Mod. Optics, vol. 39, No. 3, 569-588.

7. T. Andersen, A. Ardeberg, J. Beckers, R. Flicker, A. Gontcharov, N. C. Jessen, E. Mannery, M. Owner-Pertersen, H. Riewaldt, The proposed $50 \mathrm{~m}$ Swedish Extremely Large Telescope, 2000, Proceedings Bäckaskog Workshop on Extremely Large Telescopes, p 72.

8. R. Gilmozzi, P. Dierickx, OWL Concept study, ESO Messenger No. 100, 2000, p. 1.

9. D. Gallieni, C. del Vecchio, E. Anaclerio, P. G. Lazzirini, , LBT adaptive secondary preliminary design,, SPIE Proc. 4007, p.502, 2000.

10. E. Brunetto, F. Koch, M. Quattri, OWL: further steps in designing the telescope and in assessing its performances; SPIE Proc. 4004, 2000.

11. E. Brunetto, F. Koch, F. Biancat Marchet, M. Dimmler, Friction drive and bogies for OWL's main axes, technological step backwards or cost effective alternative?; 2002, Proc SPIE conf. on Future Giant Telescopes.

12. M. Quattri, F. Koch, L. Noethe, A. Correal Bonnet, G. Nolting, OWL wind loading characterization: a preliminary study; 2002, Proc SPIE conf. on Future Giant Telescopes.

13. R. Conan, M. Le Louarn, J. Braud, E. Fedrigo, N. Hubin, Results of AO simulations for ELTs; 2002, Proc SPIE conf. on Future Giant Telescopes.

14. E. Fedrigo, E. Marchetti, C. Arcidiacono, E. Diolaiti, Layer-oriented single and dual field of view performance for $O W L$; Proc SPIE conf. on Future Giant Telescopes.

15. A. Schumacher, L. Montoya, N. Devaney, K. Dohlen, P. Dierickx, Phasing ELTs for Adaptive Optics: preliminary results of a comparison of techniques, Proc. ESO Conf. Beyond Conventional Adaptive Optics, Venice, 2001 (http://lenin.pd.astro.it/venice2001/proceedings/).

16. L. Montoya Martinez, N. Yaitskova, P. Dierickx, K. Doehlen, Mach-Zehnder wavefront sensor for phasing of segmented telescopes; 2002, Proc SPIE conf. on Future Giant Telescopes. 
17. M. Sarazin, The New Tools for a Global Survey of Potential Sites for the Future Giant Telescopes, 2002, Proc SPIE conf. on Future Giant Telescopes.

18. P. Dierickx, B. Delabre, L. Noethe, OWL optical design, active optics and error budget; Proc.SPIE, 4003, 2000.

19. P. Dierickx, R. Gilmozzi, OWL Concept Overview; 2000, Proc. Bäckaskog Workshop on Extremely Large Telescopes, p 43.

20. P. Dierickx, Optical design and adaptive optics properties of the OWL 100-m telescope; Proc. ESO Conf. Beyond Conventional Adaptive Optics, Venice, 2001 (http://lenin.pd.astro.it/venice2001/proceedings/).

21. R. Ragazzoni, private communication.

22. N. Yaitskova, K. Dohlen, P. Dierickx, Diffraction in OWL: effects of segmentation and segments edge misfigure; 2002, Proc SPIE conf. on Future Giant Telescopes.

23. G.Chanan, M.troy, C.Ohara, "Phasing the primary mirror segments of the Keck telescopes: a comparison of different techniques", Proc.SPIE, 4003, 188-201,2000.

24. A. Schumacher,N.Devaney, L.Montoya, "Phasing segmented mirrors: a modification of the Keck narrow-band technique and its application to extremely large telescopes", Applied Optics, 41, 1297-1307, 2002.

25. J. M. Rodriguez-Ramos; Jesus J. Fuensalida,; Piston detection of a segmented mirror telescope using a curvature sensor: preliminary results with numerical simulations ; Proc. SPIE Vol. 2871, p. 613-616; 1997.

26. V. G. Orlov, S. Cuevas, F. Garfias, V. V. Voitsekhovich, L. J. Sanchez; Co-phasing of segmented mirror telescopes with curvature sensing; Proc. SPIE Vol. 4004, p. 540-551, 2000.

27. S. Esposito, N. Devaney, Segmented mirror co-phasing using Pyramid Sensor, Proc. ESO Conf. Beyond Conventional Adaptive Optics, Venice, 2001 (http://lenin.pd.astro.it/venice2001/proceedings/).

28. N. Hubin, R. Arsenault, W. Brandner, H. Bonnet, R. Conan, R. Donaldson, J. Farinato, E. Fedrigo, M. Kasper, M. Kissler, M. Le Louarn, E. Marchetti, S. Stroebele, S. Tordo; Adaptive Optics projects at ESO; 2002, Proc SPIE conf. Adaptive Optical System Technologies II.

29. J. Mariotti and G. D. Benedetto, “Pathlength stability of synthetic aperture telescopes: The case of the $25 \mathrm{~cm}$ CERGA interferometer", in Very Large Telescopes, their Instrumentation and Programs, M. H. Ulrich and K. Kjär, eds., 79, pp. 257-265, Proc. IAU colloq., 1984.

30. J. Davis, P.Lawson, A. Booth, W. Tango, and E. Thorvaldson, “Atmospheric path variations for baselines up to 80-m measured with the Sydney University stellar Interferometer", Mon. Not. R. astr. Soc. 273, pp. L53-L58, 1995.

31. R. Conan, A. Ziad, J. Borgnino, F. Martin, and A. Tokovinin, " Measurements of the wave-front outer scale at Paranal : Influence of this parameter in interferometry," in Astronomical Telescopes and Instrumentation 2000, P. Léna and A. Quirrenbach, eds., 4006, pp. 963-973, SPIE, Munich, 2000.

32. F. Martin, R. Conan, A. Tokovinin, A. Ziad, H. Trinquet, J. Borgnino, A. Agabi, and M. Sarazin, "Optical parameters relevant for High Angular Resolution at Paranal from GSM instrument and surface layer contribution," Astron. Astrophys. Suppl. Ser.”, 144, pp. 39-44, May 2000.

33. E. Marchetti, N. Hubin, E. Fedrigo, J. Brynnel, B. Delabre, R. Donaldson, F. Franza, R. Conan, M. Le Louarn, C. Cavadore, A. Balestra, D. Baade, J. L. Lizon, R. Gilmozzi, G. Monnet, R. Ragazzoni, C. Arcidiacono, A. Baruffolo, E. Diolaiti, J. Farinato, E. Viard, D. Butler, S. Hippler, A. Amorim; MAD the ESO multi-conjugate adaptive optics demonstrator; 2002, Proc SPIE conf. Adaptive Optical System Technologies II.

34. Ragazzoni R., "Pupil plane wave front sensing with an oscillating prism", J. of Mod. Opt. 43, pp. 289-293, 1996.

35. E. Vernet-Viard, R. Ragazzoni, E. Diolaiti, R. Falomo, F. Farinato, E. Fedrigo, E. Marchetti, C. Arcidiacono, A. Baruffolo, S. Esposito, M. Tordi, M. Carbillet, C. Verinaud, The Layer Oriented wavefront sensor for MAD: status and progress report; 2002, Proc SPIE conf. Adaptive Optical System Technologies II. 